\title{
POWER ALLOCATION IN COOPERATIVE SPACE-TIME CODED WIRELESS RELAY NETWORKS
}

by

Aasem N. Alyahya

Submitted in partial fulfilment of the requirements for the degree of Master of Applied Science

at

Dalhousie University

Halifax, Nova Scotia

August 2011

(c) Copyright by Aasem N. Alyahya, 2011 


\section{DALHOUSIE UNIVERSITY}

\section{ELECTRICAL AND COMPUTER ENGINEERING}

The undersigned hereby certify that they have read and recommend to the Faculty of Graduate Studies for acceptance a thesis entitled "Power Allocation in Cooperative Space-Time Coded Wireless Relay Networks" by Aasem N. Alyahya in partial fulfillment of the requirements for the degree of Master of Applied Science.

Dated: August 29, 2011

Supervisor:

Readers: 


\title{
DALHOUSIE UNIVERSITY
}

Date: August 29, 2011

\author{
Author: Aasem N. Alyahya \\ Title: $\quad$ Power Allocation in Cooperative Space-Time Coded \\ Wireless Relay Networks \\ Department: Electrical and Computer Engineering \\ Degree: M.A.Sc. Convocation: October
}

Year: 2011

Permission is herewith granted to Dalhousie University to circulate and to have copied for non-commercial purposes, at its discretion, the above title upon the request of individuals or institutions. I understand that my thesis will be electronically available to the public.

The author reserves other publication rights, and neither the thesis nor extensive extracts from it may be printed or otherwise reproduced without the author's written permission.

The author attests that permission has been obtained for the use of any copyrighted material appearing in the thesis (other than the brief excerpts requiring only proper acknowledgement in scholarly writing), and that all such use is clearly acknowledged.

Signature of Author 
To My Parents, Wife $\&$ Family 


\section{Contents}

List of Tables $\quad$ vii

List of Figures viii

Abstract $\quad$ xi

List of Abbreviations Used xii

1 Introduction 1

1.1 Propagation and Fading Laws/Broadcast Channel Characteristics . . 5

1.2 Power Control . . . . . . . . . . . . . . . . . . . . 6

1.3 Relay Networks and Cooperation Techniques . . . . . . . . . . . . . . 7

1.4 Thesis Objectives . . . . . . . . . . . . . . . . 10

1.5 Thesis Organization . . . . . . . . . . . . . . . . . . 12

2 Background $\quad 13$

2.1 BER Performance in Rayleigh Fading Channels . . . . . . . . . . . 13

2.2 Diversity . . . . . . . . . . . . . . . . 16

2.3 Space-Time Block Coding . . . . . . . . . . . . . . . . . 17

2.3.1 Alamouti's Scheme . . . . . . . . . . . . . . 18

2.3.2 Power Control for a Distributed Antenna System . . . . . . . 19

3 Multistage Relay Networks with Mean Channel Gain Information 21

3.1 Power Calculation in Random Topologies . . . . . . . . . . . . . . . . 22

3.1.1 Power Calculation for Direct Transmission . . . . . . . . . . . 23

3.1.2 Power Calculation for Store-and-Forward Transmission . . . . 24 
3.1.3 Power Calculation for STBC . . . . . . . . . . . . . . . 28

3.2 Equi-Spaced Relay Selection for Single- and Double-Stage Transmission 33

3.2.1 Single-Stage Strategy . . . . . . . . . . . . . . . . . 34

3.2 .2 Double-Stage Strategy . . . . . . . . . . . . . . . 36

3.3 Power Allocation and Optimal Positioning of Relays for STBC Relaying 39

3.3.1 Power Allocation in a Single-Stage Strategy . . . . . . . . . . 40

3.3.2 Power Allocation in a Double-Stage Strategy . . . . . . . . . . 42

4 Performance $\quad 45$

4.1 Simulation Setup . . . . . . . . . . . . . . . . 46

4.2 Excess Power Consumption . . . . . . . . . . . . . . . 47

4.3 Results with Good Channel Estimation . . . . . . . . . . . . 50

4.4 Results with Poor Channel Estimation . . . . . . . . . . . . . 57

4.5 Histograms . . . . . . . . . . . . . . . . . . 59

5 Conclusions and Future Work $\quad 61$

5.1 Thesis Contributions . . . . . . . . . . . . . . . . 61

5.2 Suggested Future Work . . . . . . . . . . . . . . . . . . . . . 64

$\begin{array}{ll}\text { Bibliography } & 66\end{array}$ 


\section{List of Tables}

3.1 Calculation of transmission power consumption for all possible paths. 26

3.2 Calculation of transmission power consumption for all possible paths. 28

4.1 Summary of the simulation results for an area of $50 \times 50 \ldots \ldots 53$ 


\section{List of Figures}

1.1 Single-hop vs direct transmission. . . . . . . . . . . . . . . 8

1.2 Double-stage cooperative relay nodes forming a MIMO channel with virtual antenna arrays. . . . . . . . . . . . . . . . . . . . 9 9

2.1 BER performance for coherent BPSK modulation with a flat Rayleigh fading channel. . . . . . . . . . . . . . . . . . 15

2.2 Alamouti STBC model. . . . . . . . . . . . . . . . . . . . . . . . . 19

3.1 Direct transmission between a source and a destination. . . . . . . . . 23

3.2 Single-stage store-and-forward transmission. . . . . . . . . . . . 24

$3.3 \quad N$ relay nodes in a wireless network. . . . . . . . . . . . 25

3.4 Double-stage store-and-forward transmission. . . . . . . . . . 26

3.5 A wireless network with 4 relay nodes. . . . . . . . . . . . 27

3.6 Transmission with a single-stage strategy using STBC. . . . . . . . . 29

3.7 Transmission with a double-stage strategy using STBC. . . . . . . . 31

3.8 A relay node located on the direct path between the source and the destination. . . . . . . . . . . . . . . . 34

3.9 Relationship between a relay node and the equi-spaced point. . . . . 35

3.10 Determining the relay node closest to the equi-spaced location. . . . . 36

3.11 Determining optimum point location for the double-stage strategy. . . 37

3.12 Relationship between the equi-spaced locations and the relay nodes. . 38

3.13 Determining the location of the optimum point in a single-stage strategy using STBC. . . . . . . . . . . . . . . . . 4 40 
3.14 Demonstrating the relationship between the relay nodes and the opti-

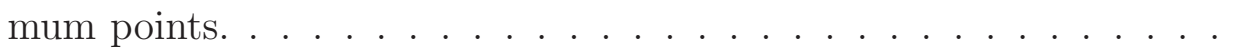

3.15 Determining the location of the optimum points in a double-stage strategy using STBC. . . . . . . . . . . . . . . . .

3.16 Transmitting data where a relay node has been selected in the first and

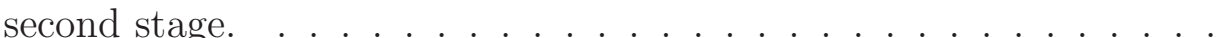

4.1 Excess power consumption in comparison with the exhaustive search method for an area of $25 \times 25$ using (a) single-stage and (b) double-stage

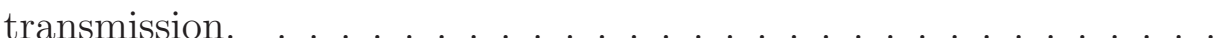

4.2 Excess power consumption in comparison with the exhaustive search method for an area of $50 \times 50$ using (a) single-stage and (b) double-stage transmission. . . . . . . . . . . . . . . . 50

4.3 Excess power consumption in comparison with the exhaustive search method for an area of $100 \times 100$ using (a) single-stage and (b) doublestage transmission. . . . . . . . . . . . . . . .

4.4 Transmission power consumption and the percentage of power savings in comparison with direct transmission for an area of $25 \times 25$, (a) \& (b) with free space propagation, (c) \& (d) with ground wave propagation, and (e) \& (f) with propagation in a dense urban environment. . . . .

4.5 Transmission power consumption and the percentage of power savings in comparison with direct transmission for an area of $50 \times 50$, (a) \& (b) with free space propagation, (c) \& (d) with ground wave propagation, and (e) \& (f) with propagation in a dense urban environment. . . . .

4.6 Transmission power consumption and the percentage of power savings in comparison with direct transmission for an area of $100 \times 100$, (a) \& (b) with free space propagation, (c) \& (d) with ground wave propagation, and (e) \& (f) with propagation in a dense urban environment.

4.7 The percentage of power savings with an incorrect estimate of propagation in a dense urban environment. . . . . . . . . . . . 
4.8 The percentage of power savings with an incorrect estimate of free space propagation. . . . . . . . . . . . . . . 58

4.9 Histogram of transmission power consumption for a single-stage strategy in a ground wave environment. . . . . . . . . . . . . . . . . . . . 60

4.10 Histogram of transmission power consumption for a double-stage strategy in a ground wave environment. . . . . . . . . . . . . . . . 60 


\section{Abstract}

Cooperative communications is a new wireless networking paradigm that allows networking nodes to collaborate through distributed transmission and signal processing to implement spatial and time signal diversity to combat the effects of fading channels. These systems exploit the wireless broadcast advantage, where transmissions from an omnidirectional antenna can be received by networking nodes that lie within its communication range. Specifically, in cooperative relaying systems the source broadcasts a message to a number of cooperative relays, which in turn resend a processed version of the information to the intended destination nodes, emulating antenna array effects. The destination nodes combine the signals received from the collaborating relays, either to increase the capacity of communication links or to increase the reliability of transmissions between the source and the destination. This is accomplished with an approach similar to that used in recently introduced spacetime coding techniques for multiple-input multiple-output (MIMO) communication systems.

This thesis is concerned with the power allocation problem in a transmit diversity wireless system with mean channel gain information. With Rayleigh fading for a given set of mean channel gains and nodes, the aim is to find the power allocation that minimizes the total transmit power for nodes involved in the transmission between the source and the destination, where all of the transmitters use single omnidirectional antennas. Specifically, the selection of store-and-forward relays deploying Alamoutitype distributed space-time coding along a multipath connection is proposed for a network with randomly distributed nodes. The proposed signal processing schemes are based on the knowledge of interdistances between nodes. By adapting the transmission power they control the selection of relay nodes, utilizing space-time coding to minimize the total energy required to transmit the information. Geometric concepts and abstractions are used to demonstrate the most energy-efficient relay choices.

The energy efficiency of conventional store-and-forward and space-time coded relaying schemes with an optimized choice of relays is analyzed for different propagation conditions and for various node densities, with potential theoretical improvements corroborated through simulations. This approach offers good power savings in comparison to the direct transmission and conventional store-and-forward approaches. In addition, the selection of relay nodes is calculated more quickly and with less complexity than is the case with the exhaustive search method. 


\title{
List of Abbreviations Used
}

\author{
AWGN Additive White Gaussian Noise \\ BER Bit Error Rate \\ BPSK Binary Phase Shift Keying \\ CSI Channel State Information \\ DF Decode-and-Forward \\ LOS Line-of-Sight \\ MATLAB Matrix Laboratory \\ MIMO Multiple-Input Multiple-Output \\ MISO Multiple-Input Single-Output \\ PSK Phase Shift Keying \\ RF Radio Frequency \\ RSSI Received Signal Strength Indicator \\ SF Store-and-Forward \\ SIMO Single-Input Multiple-Output \\ SISO Single-Input Single-Output \\ SNR Signal-to-Noise Ratio \\ STBC Space-Time Block Coding \\ Wi-Max Worldwide Interoperability for Microwave Access \\ WLAN Wireless Local Area Network \\ WN Wireless Network \\ WSN Wireless Sensor Network
}




\section{Chapter 1}

\section{Introduction}

Traditional wireless networks (WNs) have predominantly used direct point-topoint or point-to-multipoint transmissions, where the communicating nodes are equipped with a single transmitter and a single receiver deploying omnidirectional antennas. Recently this point-to-point, or single-input single-output (SISO), communication framework has been changed dramatically, with the introduction of the multipleinput multiple-output (MIMO) communication paradigm, where the source and the destination use multiple antennas at both sides of the wireless link. By transmitting in the same frequency band and at the same time, MIMO systems with advanced signal processing are greatly increasing the capacity and reliability of wireless links.

A fundamental problem in wireless network design is the allocation of limited 
resources among the network users. In cross-layer communication protocol architectures, to achieve higher bandwidth and power efficiency in wireless systems, integrated protocol designs are pursued which involve close interactions between network and physical layers [1]. In addition, to overcome distance limitations and power constraints, a natural approach in WNs is to use relay stations, with data packets taking multihop and multiple paths toward the destination [2], [3].

Cooperative communications and networking allow different nodes in a wireless network to share resources and to collaborate through distributed transmission and processing, with each node's information being sent out not only by the source but also by the collaborating nodes. Specifically, distributed wireless nodes combat the degrading effects of signal fading by automatically adapting the space-time block coding (STBC) structure to changes in the wireless propagation environment, in a manner similar to MIMO techniques. For a variety of processing algorithms and transmission protocols, performance improvements in terms of transmission rate and reliability have already been demonstrated by using the principles of the generalized STBC [4].

Cooperative techniques are being considered for a variety of wireless and mobile radio networks, including the IEEE 802 set of protocols. The IEEE 802.11 standard in the area of wireless local area networks (WLANs) for indoor environments has an IEEE 802.11s option which uses mesh networking with multihop topologies. The 
IEEE 802.16 standard, commonly referred to as Wi-Max, for wireless metropolitan area networks, has an amendment, IEEE $802.16 \mathrm{j}$, which is concerned with multihop relaying to enhance coverage, throughput, and system capacity. Multihop cooperative strategies are also being proposed for cellular systems with femto-sized cells as well as for a broad range of wireless sensor networks (WSNs) and ad hoc networks [5], [6].

In most of the work on cooperative relaying, wireless system models do not fully investigate the wireless broadcast advantage, where nodes overhearing the signals can be exploited to achieve much better tradeoffs between the target bit error rate (BER) performance and the required transmit power. This is a motivation for the research in this thesis, where additional design options are considered for energy-efficient wireless network operation. Specifically, the design of routing protocols with multiple-stage relays utilizing STBC along a data path is proposed for a wireless network with randomly distributed nodes. This work exploits the fact that modern wireless equipment can be designed to adjust power levels automatically to the minimum needed to achieve the desired target BER, by varying the output power depending upon channel state information (CSI) [7]-[11].

The power control proposed in this thesis for the topology formation of the relay nodes involved in STBC considers minimization of the total power in a single- and double-stage multihop type of wireless networks. To achieve energy-efficient performance, the nodes adjust their transmission power to overcome deterministic path loss 
and to reach the intermediate relays using a distributed type of Alamouti STBC with sufficient receive power to ensure reliable communication. The decisions about which nodes are involved in forwarding the STBC data between the source and destination pairs are based only on the distances between the nodes, which in turn are linked to the received signal strength indicator (RSSI) values and the transmit power used to reach the desired nodes in the network [12]. In addition to addressing the geometric analysis of the power-efficient topology problem, this thesis also focuses on finding computationally efficient algorithms for the selection of STBC relay nodes which will result in a topology formation requiring close to minimum energy. The significance of this work is to demonstrate how to use the wireless broadcast advantage in the integration of STBC cooperative communications and adaptive power control for more efficient transmit power utilization by exploiting information about the channel states and user positions in the network. In this case, more efficient use of WN node batteries comes at the expense of more complex signal processing at the nodes, as well as the overhead associated with learning the CSI in a form of mean channel gain information as determined by the RSSI and deterministic signal attenuation with distance.

The design of WNs is a complex field involving the knowledge and understanding of many different areas, including radio channel characteristics, transceiver design in fading environments, and communication protocol implementation. Some of these issues are examined in the following sections, and a more detailed background is 
provided in Chapter 2.

\subsection{Propagation and Fading Laws/Broadcast Chan- nel Characteristics}

When the wireless signal is transmitted from the transmitter to the receiver, it undergoes through many impairments such as attenuation, fading, time and frequency dispersion, non-linear distortions, etc. This thesis considers only two causes: free space loss and multipath fading.

Any transmitted signal will fade and weaken as it travels through the air, with the result that when the signal arrives at the receiver, it will be received with less energy than was present at the transmitter side. As a first approximation, the signal power at the receiver decreases with increasing distance between the transmitter and receiver. This means that the transmitter should utilize sufficient power when sending the signal to ensure that the signal is received with an acceptable power level [13], [14].

Wireless networks are usually placed in areas where many obstacles exist. If a signal runs into obstacles, reflections of the signal with different phase shifts result. From a single transmission, the receiver therefore receives multiple signals with different power levels. In the case of Rician fading, among many reflections there is a line-of-sight signal, which should have the highest power level. If no line-of-sight signal exists and the signal is retrieved from the combined effects of reflections, this can be modeled as Rayleigh fading [15], [16]. 


\subsection{Power Control}

This section presents the formula that is used in this thesis to calculate the transmission power. From the deterministic path loss model it is known that the received power is a decreasing function of distance, which can be written as follows:

$$
P_{R x}=\frac{P_{0}}{r^{\alpha}}
$$

where $P_{0}$ is the reference power at a distance equal to 1 , which is dependent upon the transmission power, the frequency used and the transmitter and receiver antenna gain; $r$ is the distance traveled by the signal from the transmitter to the receiver; and $\alpha$ is the path loss exponent. The path loss exponent, $\alpha$, which depends upon the surrounding environment and terrain structure, can have a value ranging from 2 in free space environments to 6 in dense urban areas [17].

In this thesis it is assumed that the power of noise at the receiver is $4 \mathrm{~mW}$; in practical interpretations of the thesis simulation results an appropriate scaling power should be applied. The target BER for this research is $10^{-3}$. To achieve this with Rayleigh fading, the signal-to-noise ratio (SNR) should be $24 d B$ for conventional transmissions and $14 d B$ for cooperative relay transmissions with two antennas. Further information about the SNR is presented in the following chapter. With these assumptions, in order to meet the required $B E R=10^{-3}$ in a SISO system, the received signal power is $1 W$, and with some algebraic manipulations of (1.1), the 
transmit power $P_{T x}$ can be written as follows:

$$
P_{T x} \sim r^{\alpha}
$$

In this thesis, omnidirectional antennas and homogenous propagation conditions are assumed. These assumption simplify the notations in this thesis.

\subsection{Relay Networks and Cooperation Techniques}

In a cooperative paradigm, nodes which would have remained silent in a traditional point-to-point communication framework cooperate with the source and the destination to increase communication capacity and reliability. Initial work on cooperative communications introduced the concept of the relay node, where different relays receive noisy copies of the same information and, in a store-and-forward operation, each relay decodes the original information and forwards it toward the destination. This process reduces the energy required to send the information from the source to the destination, as illustrated in Figure 1.1, where two-hop (single-stage) transmission via node $R$ is compared to direct transmission from the source $S$ to the destination $D[18]$

By using basic geometry, from Figure 1.1, $d^{2}=d_{1}^{2}+d_{2}^{2}-2 d_{1} d_{2} \cos \gamma$. Thus, if $R$ lies in the circle $\mathcal{C}$, implying $\cos \gamma \leq 0$, it can be seen that $d^{2} \geq d_{1}^{2}+{ }_{2}^{2}$. This implies that in a free space propagation environment where $\alpha=2$, the total power required to 


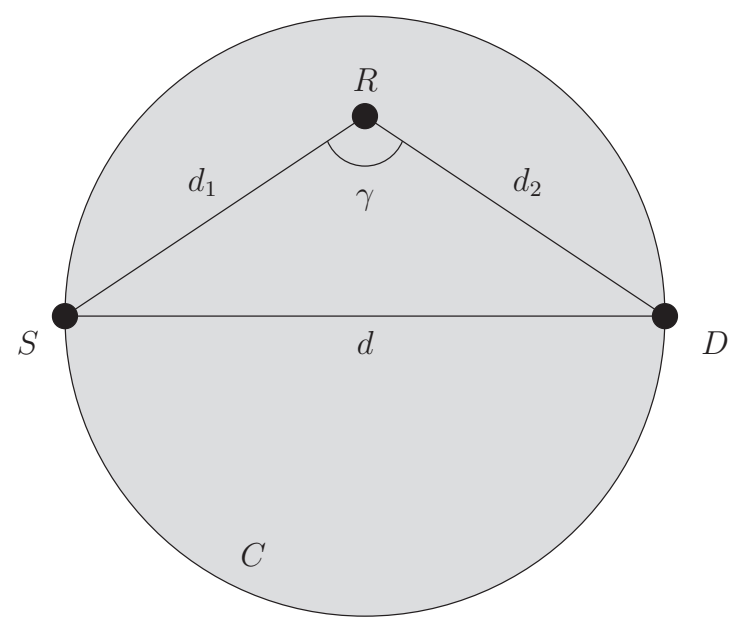

Figure 1.1: Single-hop vs direct transmission.

transmit the information from the source to the destination using direct transmission, $P^{D}=d^{2}$ is greater than the power used in store-and-forward mode via relay $R$, which is $P^{S F}=d_{1}^{2}+d_{2}^{2}$.

From the point of view of energy consumption, it is better to communicate using short multihop paths between the transmitter and receiver, with reduced transmission power at each node, rather than using high power levels at the transmitter so that the signal is received directly at the destination. The nodes could even lower their transmission power more if additional stages are used, which results in more total power savings.

More recent work on cooperative communications treats the wireless channel as a MIMO channel with space-time coding based on cooperative diversity algorithms. In wireless networks, signal fading arising from multipath propagation is usually combated through the use of diversity transmission of redundant signals over essentially 
independent channel realizations, in conjunction with suitable receivers combining multiple received signals and averaging the channel effects. Space, or multipleantenna, diversity techniques are particularly attractive as they offer dramatic performance gains. In contrast to the more conventional forms of single-user space diversity with physically co-located antenna elements, the distributed space-time coded protocols in cooperative communications build upon the classical relay channel using nodes equipped with single element antennas which act as the distributed virtual antenna array visualized in Figure 1.2 [19].

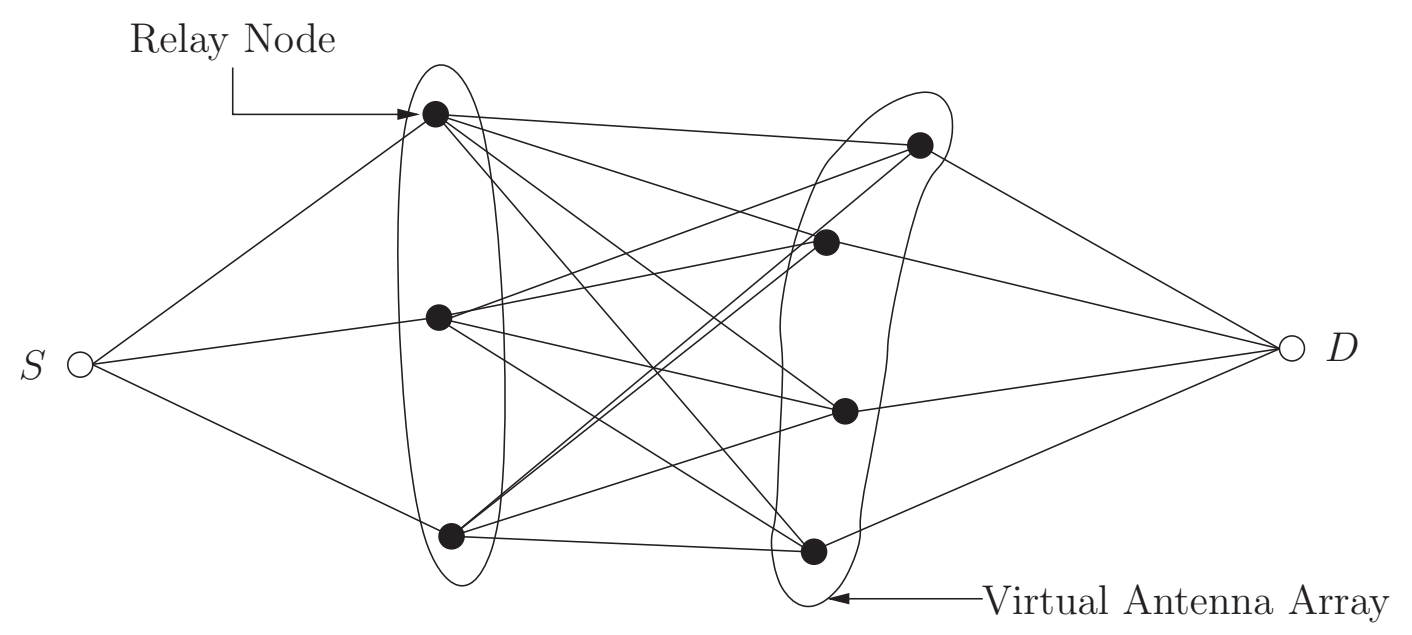

Figure 1.2: Double-stage cooperative relay nodes forming a MIMO channel with virtual antenna arrays.

Figure 1.2 illustrates an assembly of spatially distributed and possibly cooperating terminals forming a distributed MIMO channel by means of virtual antenna arrays. 


\subsection{Thesis Objectives}

The objective of this study is to find a good routing algorithm and a power allocation scheme that uses the transmission power of nodes involved in data communication between the source and the destination as the performance metric. The investigations focus on two types of relay strategy: (i) the single-stage relay strategy and (ii) the double-stage relay strategy. The motivating principle in these investigations is to find the WN nodes which, when STBC is deployed, will minimize transmission power consumption. This thesis takes advantage of: (i) cooperative relay nodes deploying STBC and (ii) selection of the optimum forwarding topology among a number of different options obtained through adjusting the transmission power level. The contribution of this thesis is in the integration of these two approaches, which until now have been investigated only as separate solutions. With the utilization of distributed STBC and adaptive power allocation, greater power savings are realized than are achieved with the conventional store-and-forward approach. This is due to the exceptional STBC performance in fading channels. Direct transmission and store-and-forward transmission are used as benchmarks in this study when comparing the results for the proposed topology and power.

This thesis uses a distributed routing system similar to the link-state routing protocol in a wireline network. In such a wireline routing protocol, all nodes in the network are aware of the complete topology. In this thesis an equivalent approach 
is assumed, where the interdistance information is distributed among all the nodes in the WN. The distribution of information in the WN could be done in a manner similar to the link-state routing protocol. The goal of the thesis is to find an efficient algorithm with reduced processing complexity that will determine the best routing path, with knowledge of the transmission power. In order to introduce an algorithm similar to that used in the link-state protocol, the focus is on developing an equivalent to Dijkstra's algorithm. In a wireline network this algorithm is very efficient due to the limited number of links between the nodes. It therefore calculates the routing path from a source to a destination in a short amount of time. However, this algorithm would not have the same efficiency when applied to an ad-hoc WN, because it uses a mesh topology which results in greater complexity when calculating the routing path [20]. The algorithm process time would increase even more when STBC is deployed, as this results in more possible paths from which to select.

Simulations in MATLAB are the primary vehicle used to verify the performance of the proposed selection approach. In the simulations, the positions of the source and the destination are fixed and randomly distributed potential relay nodes are generated. By utilizing the knowledge of the distances between all the nodes, the transmission power consumption is calculated in different propagation conditions for different numbers of relay nodes and sizes of wireless network space. Different topologies with distributed STBC are obtained by adjusting the power, and the corresponding power 
is calculated. The most energy-efficient topology could be selected by means of an exhaustive search, however, in this thesis the computational complexity is reduced by demonstrating an efficient method of obtaining the power and relay choice.

\subsection{Thesis Organization}

This thesis is organized as follows: Chapter 2 provides an overview of the BER performance in Rayleigh fading channels and introduces the Alamouti version of spacetime block coding. Chapter 3 describes how to select the relay nodes to determine the path for routing the information to the destination with minimum power consumption. Chapter 4 presents the results and the performance of the proposed relay node selection approach. Finally, in Chapter 5 the thesis results are summarized and suggestions are provided for future work. 


\section{Chapter 2}

\section{Background}

In this chapter the performance of wireless systems in fading environments and the methods of mitigating BER degradation are reviewed. Specifically, the application of space-time block coding is discussed, and it is shown how diversity reduces the total transmit power.

This chapter is organized as follows. In Section 2.1 the Rayleigh fading channel is explained. Diversity techniques are then discussed in Section 2.2. Finally, the space-time block coding method is introduced in Section 2.3.

\subsection{BER Performance in Rayleigh Fading Chan- nels}

Additive white Gaussian noise (AWGN) is the channel model most commonly used in the analysis of communication links. This model considers additive effects of 
random interference which, due to the central limit theorem, are well characterized by the Gaussian random variable. This AWGN is omnipresent in all communication transceivers in the form of thermal noise. Another type of interference that may affect a wireless signal is fading. This results from multipath propagation in radio channels and is modeled as a multiplicative effect. The problem is that fading introduces variability in the instantaneous received SNR which, in the AWGN model, is fixed. The variability in the instantaneous SNR in fading channels causes variability in the BER at the receiver; when this instantaneous BER is averaged over the distribution of the instantaneous SNR, the averaged BER performance in fading environments is much poorer in fading channels than with AWGN alone for the given average SNR in both models. There are two types of fading: shadowing at the macro level and fast fading at the micro level. This thesis is concerned only with the latter. For fast fading, two types of model can be used: the Rician fading channel and the Rayleigh fading channel [21]- [23]. In this thesis the Rayleigh fading channel is considered because it is assumed that there is no dominant line-of-sight signal.

Figure 2.1 shows the (average) BER performance as a function of the (average) SNR for coherent binary phase shift keying (BPSK) signaling. The green and blue curves indicate the performance with AWGN and Rayleigh fading, respectively, where the transmitter and receiver use single antennas (SISO model). The red curve shows 
the performance with Rayleigh fading in the same system when two transmit antennas are deployed, in configurations discussed in the following two sections. From Figure 2.1 it can be seen that to maintain a BER of $10^{-3}$, the Rayleigh model requires an average signal-to-noise ratio increase of approximately $17 d B$ in comparison to the AWGN model. This difference between the two models increases as the target BER decreases. A simple solution to maintain the same BER performance would be to increase the transmission power to overcome fading. However, this is not always possible because wireless transceivers are usually powered by a finite energy source: the battery.

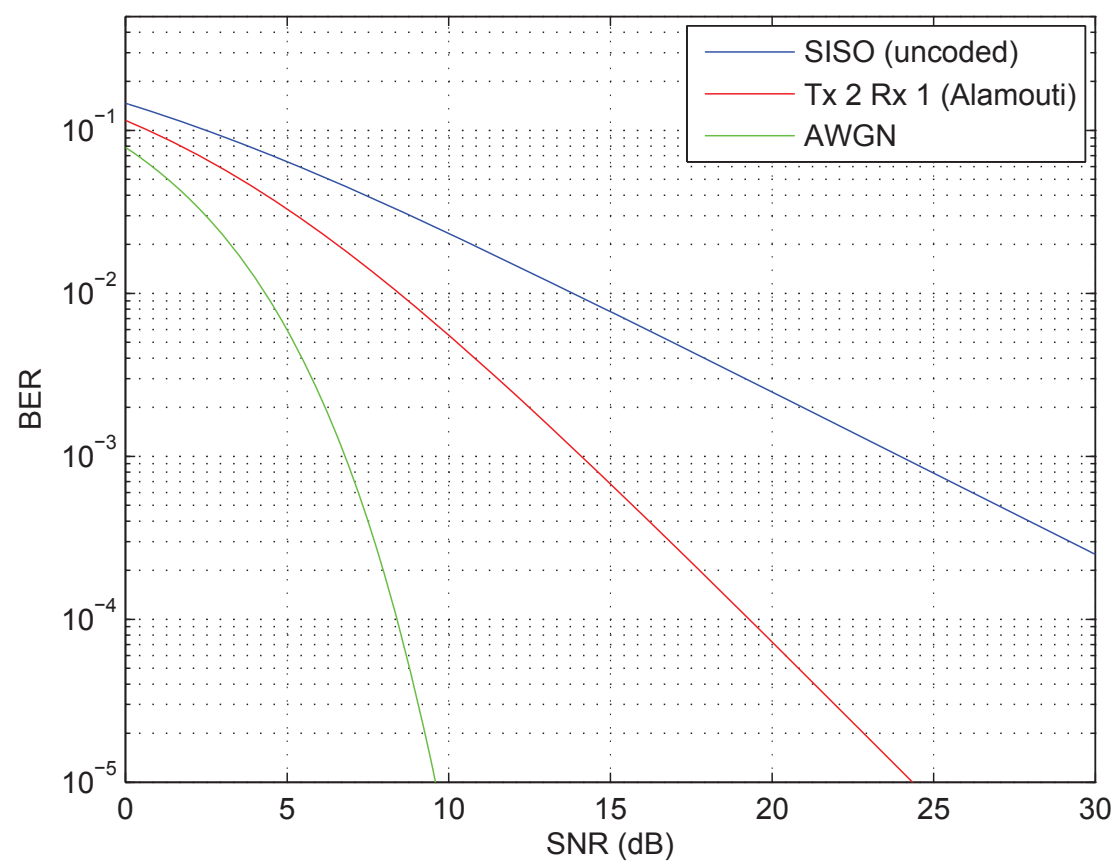

Figure 2.1: BER performance for coherent BPSK modulation with a flat Rayleigh fading channel. 


\subsection{Diversity}

The best solution for overcoming BER performance degradation in fading environments is the use of diversity. The space diversity technique provides for sending multiple replicas of the information on different paths. Diversity relies on the principle that when fading occurs (resulting in a weaker received signal), it will affect only some of the redundant paths, so that some of the paths have severe fading while others have less attenuation. The use of different domains to separate the redundant signals results in different types of diversity: frequency, time and space diversity [24].

In the case of frequency diversity, different frequencies are used to send the same information, thus providing a fully redundant logical path. The frequency separation between the different logical paths must be enough to ensure independent fading effects for each frequency. However, using this method to achieve diversity involves sacrificing bandwidth, which is a scarce resource.

Another method is time diversity, where replicas of the message are sent in different time slots. Error control coding is combined with time interleaving to achieve time diversity. The error control coding carries the replicas of the information, and time interleaving creates time separation between the two redundant data transmissions. The disadvantage of this technique is that it reduces the throughput and causes a delay in the decoding of data.

Space diversity is implemented by using an array of antennas arranged in space 
to transmit and/or receive multiple versions of the same signal along different physical paths. To ensure good performance improvement, the multiple antennas should be physically separated so that fading is independent for each physical path. The required separation distance is usually affected by the frequency, the propagation environment and the antenna height. Space diversity is the preferred diversity scheme because it does not use the limited resources of time or frequency, and it requires only investment in antennas and signal processing hardware. The three main system models which use space diversity are multiple-input multiple-output (MIMO), which uses multiple transmitters and receivers; single-input multiple-output (SIMO), which uses a single transmitter and multiple receivers; and multiple-input single-output (MISO), which uses multiple transmitters and a single receiver[25].

As described above, there are three types of diversity that can be used to mitigate the fading problem. However, hybrids of these approaches can also be implemented, by combining some of these primary methods. For example space-time block coding, described in more detail in the following section, integrates space and time diversity.

\subsection{Space-Time Block Coding}

Space-time block coding (STBC) is a diversity technique that combines space and time redundancy to reduce the deterioration caused by fading. In this section Alamouti's scheme is presented [26], which is a STBC model used in this thesis. This 
is followed by a description of the transmission power savings used for STBC.

\subsubsection{Alamouti's Scheme}

Alamouti's scheme was the first STBC to provide a full diversity system with two transmit antennas. Many types of diversity model have been created as variants of this basic scheme, however here only the MISO version is considered. This consists of two transmitters and one receiver, as shown in Figure 2.2. As can be seen in the figure, the information is represented by two M-ary symbols, $s_{1}$ and $s_{2}$. The encoder takes a block of the two modulated signals $s_{1}$ and $s_{2}$ and sends them to the antennas with in the same frequency band, according to the following matrix:

$$
S=\left[\begin{array}{cc}
s_{1} & -s_{2}^{*} \\
s_{2} & s_{1}^{*}
\end{array}\right]
$$

where each column represents the same time slot for one symbol, and each row represents data being sent from the same antenna. Thus, in the first time slot, the first antenna sends symbol $s_{1}$ while the second antenna is sending symbol $s_{2}$. In the second time slot, the first antenna sends $-s_{2}^{*}$ while the second antenna is sending $s_{1}^{*}$, where * refers to the complex conjugate of the original signal. The signals in the same time slot should be synchronized and sent together so that they will be received at the same time by the receiver. At the receiver side the decoder separates the two symbols by using the two signals received from the different time slots. For the decoder, the 

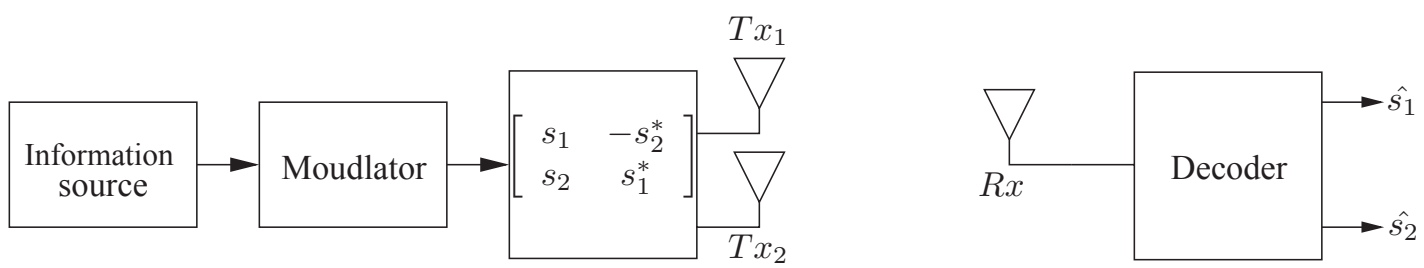

Figure 2.2: Alamouti STBC model.

basic components of Alamouti's scheme are the channel estimator and the maximum likelihood detector [27]. This scheme implements space diversity of the order $L=2$ at the transmitter and permits a dramatic reduction of the transmit power required in SISO systems with Rayleigh fading to achieve a given BER. The BER performance of Alamouti's scheme is indicated by the red curve in Figure 2.1.

\subsubsection{Power Control for a Distributed Antenna System}

As described in the previous section, in the Alamouti version of STBC, the transmitter deploys two antennas. This section discusses how to calculate the transmission power per antenna so as to achieve the target BER at the receiver. In Section 1.2 a transmission power equation was presented that considers a model using only a single transmitter and a single receiver, referred to as a single-input single-output (SISO) system. From Figure 2.1, it can be seen that to maintain $B E R=10^{-3}$ in a Rayleigh fading environment, the difference between the signal-to-noise ratios for the SISO and MISO systems is $10 d B$. Thus, when sending a signal with STBC the transmission power required can be reduced by an amount equal to the difference between the two 
signal-to-noise ratios. From (1.2) it is possible to arrive at the following relation:

$$
P_{T x}=G \cdot r^{\alpha}
$$

where $P_{T x}$ is the transmission power per antenna and $\mathrm{G}$ is the scaling factor, for scaling between the coded and uncoded signal-to-noise ratios. The scaling factor can be calculated by using the following equation:

$$
G=\frac{S N R_{S T B C}}{S N R_{S I S O}}
$$

Here $S N R_{S T B C}$ is the signal-to-noise ratio for the STBC and $S N R_{S I S O}$ is the uncoded signal-to-noise ratio (both on the linear scale). For example, for a target of $B E R=$ $10^{-3}$, from Figure 2.1 it can be found that $S N R_{S T B C}=14 \mathrm{~dB}$ and $S N R_{S I S O}=24 \mathrm{~dB}$; then the scaling factor is equal to 0.1. Thus, if STBC is used to transmit a signal, only $10 \%$ of the transmission power is required per antenna, in comparison to a SISO model. Since two antennas are used, this results in $80 \%$ power savings. 


\section{Chapter 3}

\section{Multistage Relay Networks with Mean Channel Gain Information}

In this chapter, three methods of transmitting data between a single source and a single destination are investigated: (i) direct transmission, (ii) store-and-forward transmission with multiple relays, and (iii) multistage distributed STBC. The first method, direct transmission, is single-hop communication which is used as a reference for comparing the total transmit power required by different methods. The second method, store-and-forward transmission, is a conventional multi-hop transmission where some nodes act as relays. For this method the selection of relay nodes will be discussed, with the corresponding power allocations that offer savings in the transmit power required. The third method is multistage relaying using STBC. Motivated by the store-and-forward approach, a new solution will be proposed for selecting the relay nodes when distributed STBC is utilized. With this approach, it is anticipated that selecting appropriate relay nodes in this computationally efficient scheme 
will minimize the power consumption, with savings comparable to those achieved by exhaustive search methods over all possible redundant paths.

This chapter is organized as follows: First, in Section 3.1 the three methods are presented and basic power transmission calculations are provided for each of them. Node selection using store-and-forward for single- and double-stage transmission is then explained in Section 3.2. Finally, the proposed approach for relay node selection for both single- and double-stage strategies using STBC is discussed in Section 3.3.

\subsection{Power Calculation in Random Topologies}

This section first introduces the direct transmission method, followed by the multistage store-and-forward method, and concluding with multistage STBC. All three methods are described by showing how a signal is transmitted and how much transmit power is required to deliver the data reliably from the source to the destination via different redundant paths. An explanation is also provided of how many different node configurations for the forwarding process are available in a wireless network, in the case of different modes of operation. The number of different topologies found in the power allocation will determine the complexity of the exhaustive search used to find the topology which minimizes the total transmit power required. 


\subsubsection{Power Calculation for Direct Transmission}

First direct transmission from a single source to a single destination is considered. Figure 3.1 shows direct transmission from a source to a destination, where $r_{S D}$ is the distance between the source and the destination. It is assumed that the source can communicate directly with the destination by using direct, i.e., one-hop transmission. From Section 1.2, it can be seen that the transmit power required to send a signal from one of these nodes to the other is:

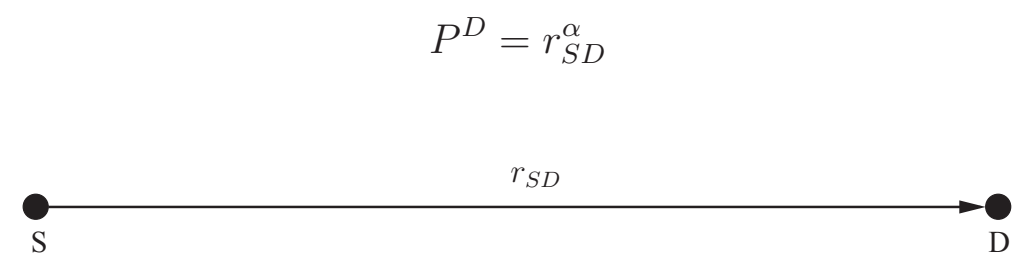

Figure 3.1: Direct transmission between a source and a destination.

It should be noted that the transmission power required increases rapidly as the distance and the attenuation exponent $\alpha$ increase. Extensive research has been carried out in order to reduce this power requirement at the system level. One solution is to send the information first to a relay node and then to the destination, so that the shorter transmission distances reduce the total power required; this is discussed in the following sections. 


\subsubsection{Power Calculation for Store-and-Forward Transmission}

To overcome the attenuation effects associated with increased distance, store-andforward is used where relay nodes are utilized in order to minimize the total power required to deliver the data to its destination.

\subsubsection{Single-Stage Strategy}

A single-stage strategy is considered where only one relay nodes is used. As shown in Figure 3.2, the transmission from the source to the destination is divided into two phases. $R_{1}$ represents a randomly located relay node. It is assumed that distance $r_{S 1}$ between the source and the relay node and distance $r_{1 D}$ between the relay node and the destination are known.

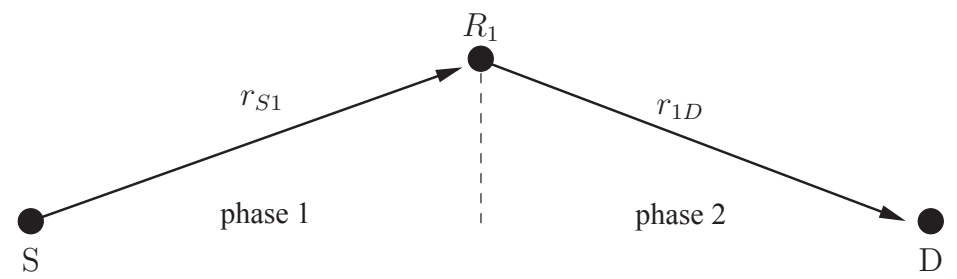

Figure 3.2: Single-stage store-and-forward transmission.

In the first phase, the information is sent from the source to the relay node. From Section 1.2 , it can be seen that the power required to achieve reliable transmission to a relay node is $P_{1}=r_{S 1}^{\alpha}$. In the second phase, the information is sent from the relay node to the destination. The same equation is used to calculate the power consumption, except that the distance $r_{1 D}$ is used instead of $r_{S 1}$. Thus the total 
transmit power required to send the information from the source to the destination is the sum of the transmit power required for the two phases:

$$
P^{S-S F}=r_{S 1}^{\alpha}+r_{1 D}^{\alpha}
$$

Next a wireless network will be considered which consists of a single source, a single destination and $N$ nodes each potentially serving as a relay. In this thesis it is always assumed that the source and the destination have fixed positions and that the $N$ nodes which could be used as relays are randomly distributed in a rectangular area. It is also assumed that the distances from the source and the destination to the nodes are known and that the distances between the nodes are also known. In addition, it is assumed that the path loss exponent $\alpha$ is known and is the same for all communications in the network. Thus, to implement a single-stage strategy using store-and-forward transmission, there could be $N$ possible paths as illustrated in Figure 3.3. Table 3.1 shows the power consumption required for each path.

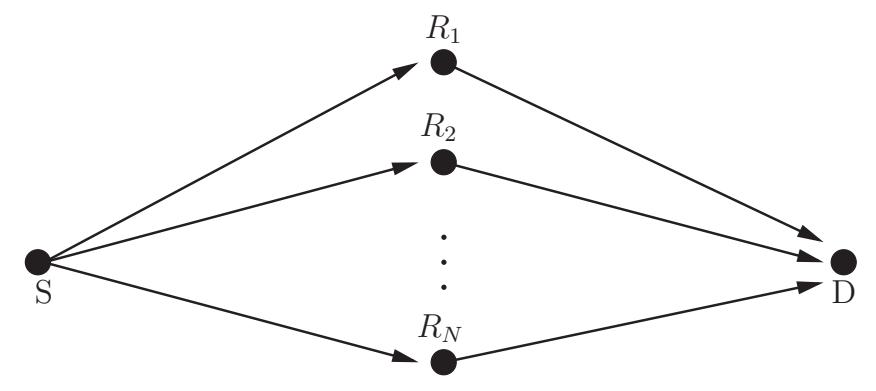

Figure 3.3: $N$ relay nodes in a wireless network.

In order to select the relay in conventional store-and-forward transmission, the node associated with the lowest total transmit power is found in Table 3.1. 
Table 3.1: Calculation of transmission power consumption for all possible paths.

\begin{tabular}{|c|c|}
\hline $\begin{array}{c}\text { Relay } \\
\text { node }\end{array}$ & $\begin{array}{c}\text { Transmission } \\
\text { power }\end{array}$ \\
\hline 1 & $P_{1}^{S-S F}=r_{S 1}^{\alpha}+r_{1 D}^{\alpha}$ \\
\hline 2 & $P_{2}^{S-S F}=r_{S 2}^{\alpha}+r_{2 D}^{\alpha}$ \\
\hline 3 & $P_{3}^{S-S F}=r_{S 3}^{\alpha}+r_{3 D}^{\alpha}$ \\
\hline$\vdots$ & $\vdots$ \\
\hline $\mathrm{N}$ & $P_{N}^{S-S F}=r_{S N}^{\alpha}+r_{N D}^{\alpha}$ \\
\hline
\end{tabular}

\subsubsection{Double-Stage Strategy}

In this strategy, two relay nodes are used. When an extra relay node is added, the distance traveled by the signal in a single phase is less than is the case with a single-stage strategy. From this it is anticipated that additional power savings will be achieved.

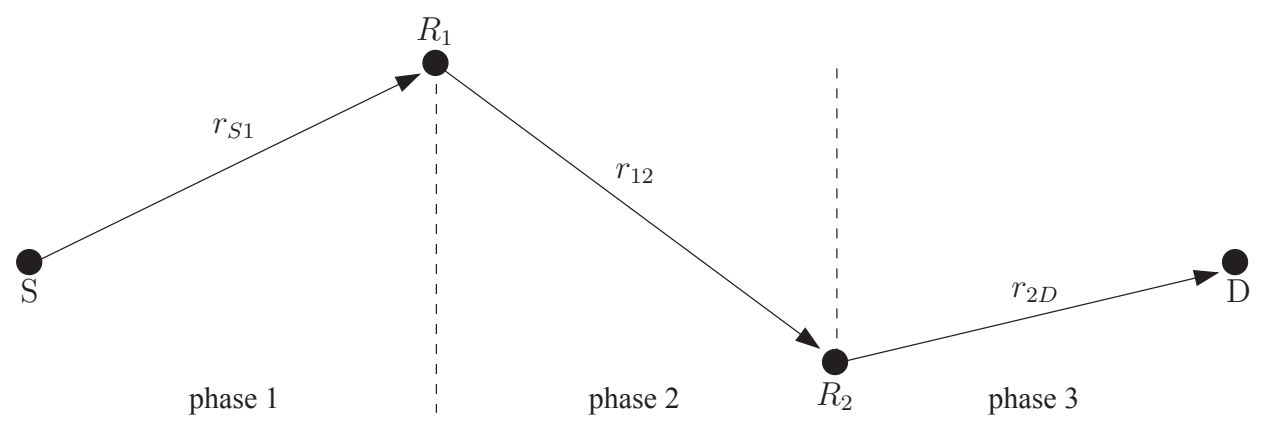

Figure 3.4: Double-stage store-and-forward transmission.

Figure 3.4 will now be considered, where $R_{1}$ and $R_{2}$ represent the first and second relay nodes respectively. The data are transmitted in three sequential phases. In the first phase the data are sent from the source to the first relay node. The calculation of the required transmission power is the same as in the single-stage strategy: $P_{1}=r_{S 1}^{\alpha}$, 
where $r_{S 1}$ is the distance between the source and the first relay node. In the second phase the signal is transmitted from $R_{1}$ to $R_{2}$ and the transmission power required is $P_{2}=r_{12}^{\alpha}$, where $r_{12}$ is the distance between the two relay nodes $R_{1}$ and $R_{2}$. In the third phase, the signal is sent from $R_{2}$ to the destination, and the transmission power required is $P_{3}=r_{2 D}^{\alpha}$. Thus, the total transmission power required to transmit the signal from the source to the destination using the double relay strategy is the sum of the power required for the three phases:

$$
P^{D-S F}=r_{S 1}^{\alpha}+r_{12}^{\alpha}+r_{2 D}^{\alpha}
$$

If it is assumed that there are $N$ randomly distributed relay nodes available in a wireless network, for $N$ relay nodes there would be $N \cdot(N-1)$ possible paths from which to select. For example, Figure 3.5 illustrates a source, a destination and four randomly distributed relay nodes. In Table 3.2, all of the 12 possible paths have been considered, and the total transmit power for each path is shown. The figure illustrates only the three paths shown in the first row of the table.

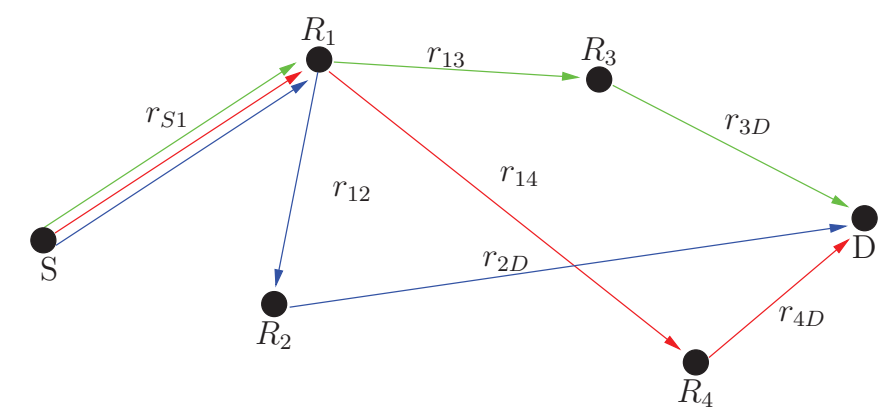

Figure 3.5: A wireless network with 4 relay nodes. 
Table 3.2: Calculation of transmission power consumption for all possible paths.

\begin{tabular}{|c|c|c|c|c|}
\hline First & \multicolumn{4}{|c|}{ Second Stage } \\
Stage & $R_{1}$ & $R_{2}$ & $R_{3}$ & $R_{4}$ \\
\hline$R_{1}$ & & $r_{S 1}^{\alpha}+r_{12}^{\alpha}+r_{2 D}^{\alpha}$ & $r_{S 1}^{\alpha}+r_{13}^{\alpha}+r_{3 D}^{\alpha}$ & $r_{S 1}^{\alpha}+r_{14}^{\alpha}+r_{4 D}^{\alpha}$ \\
\hline$R_{2}$ & $r_{S 2}^{\alpha}+r_{21}^{\alpha}+r_{1 D}^{\alpha}$ & & $r_{S 2}^{\alpha}+r_{23}^{\alpha}+r_{3 D}^{\alpha}$ & $r_{S 2}^{\alpha}+r_{24}^{\alpha}+r_{4 D}^{\alpha}$ \\
\hline$R_{3}$ & $r_{S 3}^{\alpha}+r_{31}^{\alpha}+r_{1 D}^{\alpha}$ & $r_{S 3}^{\alpha}+r_{32}^{\alpha}+r_{2 D}^{\alpha}$ & & $r_{S 3}^{\alpha}+r_{34}^{\alpha}+r_{4 D}^{\alpha}$ \\
\hline$R_{4}$ & $r_{S 4}^{\alpha}+r_{41}^{\alpha}+r_{1 D}^{\alpha}$ & $r_{S 4}^{\alpha}+r_{42}^{\alpha}+r_{2 D}^{\alpha}$ & $r_{S 4}^{\alpha}+r_{43}^{\alpha}+r_{3 D}^{\alpha}$ & \\
\hline
\end{tabular}

\subsubsection{Power Calculation for STBC}

Use of the multistage store-and-forward method, described in the previous section, reduces the transmit power required through the strategy of dividing the distance to be covered, so as to overcome the effects of deterministic signal attenuation. To decrease performance degradation effects due to Rayleigh fading, a distributed STBC approach will be deployed, where the wireless network nodes form a virtual antenna array. In this thesis the MISO model is used to send the information from two transmitters to a single receiver in accordance with Alamouti's scheme. Two cooperative nodes relaying the same data from the source are used to send the information to the destination in a manner similar to Alamouti's original scheme, where two-element antennas send data from a single source. The difference is that in the present cooperative scheme the antennas sending the STBC data to the destination are not co-located. 


\subsubsection{Single-Stage Strategy}

In this section a single-stage strategy with distrusted STBC is investigate first. Figure 3.6 represents a wireless network that contains a source, a destination and two cooperative relay nodes, $R_{1}$ and $R_{2}$. Transmission from the source to the destination is divided into two phases.

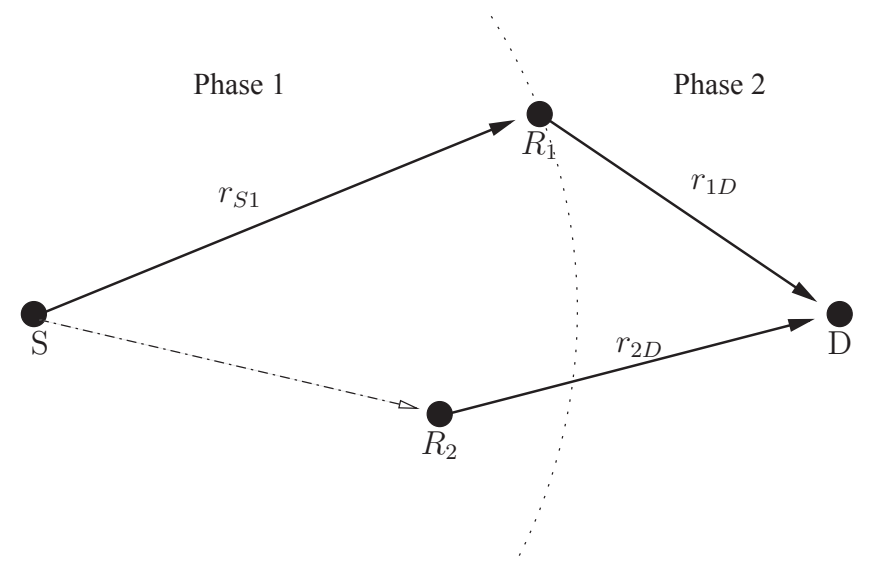

Figure 3.6: Transmission with a single-stage strategy using STBC.

The first phase involves transmission from the source to the relay nodes $R_{1}$ and $R_{2}$. In order to save power and time, the transmitted signal is broadcast to both nodes at the same time instead of sending it in separate transmissions. The transmit power used for this broadcast should ensure that both relay nodes receive the information reliably. This is achieved by sending the information with sufficient transmission power to reach reliably the farther relay node of the two. For the first phase, this power can be represented as:

$$
P_{1}=\max \left(r_{S 1}^{\alpha}, r_{S 2}^{\alpha}\right)
$$


In the next phase the two relay nodes $R_{1}$ and $R_{2}$ are used to send the information to the destination simultaneously. From (2.3) the required transmission power per antenna is known. To calculate the total transmission power for both antennas, it can be concluded that the power required in the second phase is:

$$
P_{2}=G \cdot r_{1 D}^{\alpha}+G \cdot r_{2 D}^{\alpha}
$$

One thing which must be ensured is that in the second phase the transmitted signals from the two cooperative nodes should have the same average energy level at the receiving side.

Finally, the sum of the transmission power required for the two phases is calculated to arrive at the total transmission power:

$$
P^{S-S T B C}=\max \left(r_{S 1}^{\alpha}, r_{S 2}^{\alpha}\right)+G \cdot r_{1 D}^{\alpha}+G \cdot r_{2 D}^{\alpha}
$$

In the case of a wireless network with $N$ available randomly distributed relay

nodes, there would be $\left(\begin{array}{c}N \\ 2\end{array}\right)=\frac{N \cdot(N-1)}{2}$ possible paths, where $\left(\begin{array}{l}n \\ k\end{array}\right)$ represents the number of $k$ element subsets in a set of $n$ elements. For example, in Figure 3.5 there are 4 relay nodes, which yield 6 possible paths.

\subsubsection{Double-Stage Strategy}

Here a double-stage strategy using STBC is described. It has been seen that for a single-stage strategy with STBC, two relay nodes are used. Thus, in the double-stage strategy two relay nodes are added to represent the second stage. 
As illustrated in Figure 3.7, there is a source, $S$; a destination, $D$; and four randomly distributed relay nodes, $R_{i}$, where $i \in 1,2,3,4$. $R_{1}$ and $R_{2}$ represent the first stage, and $R_{3}$ and $R_{4}$ represent the second stage. In this strategy the transmission is divided into four phases.

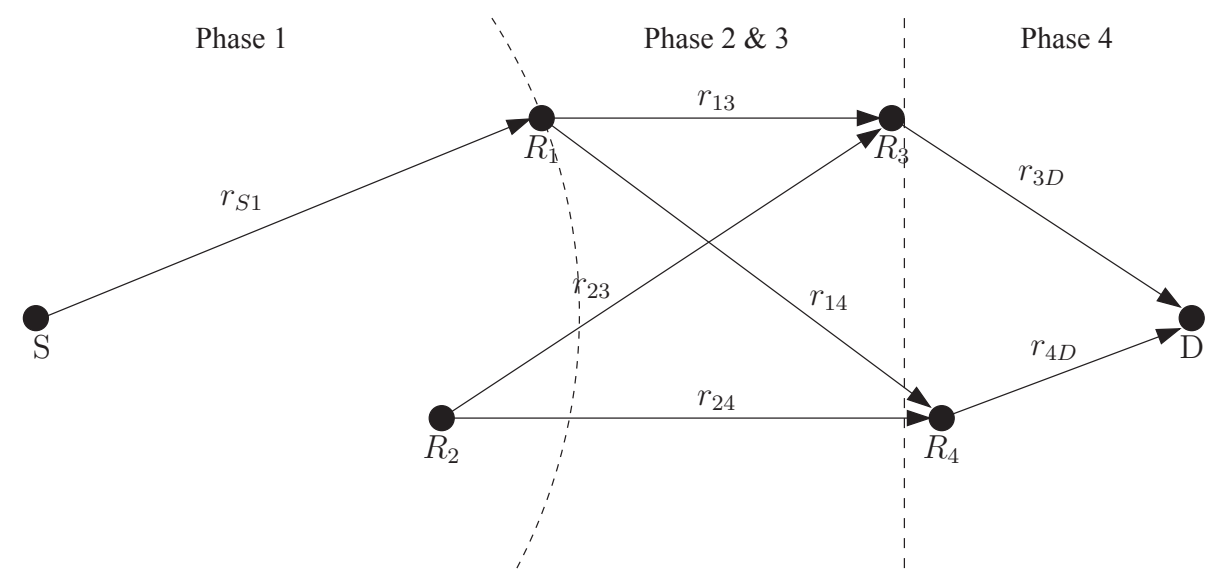

Figure 3.7: Transmission with a double-stage strategy using STBC.

The first phase is the same as in the single-stage strategy, where the source broadcasts the information to the first stage (nodes $R_{1}$ and $R_{2}$ ). The transmit power used for this broadcast should ensure that both relay nodes receive the information reliably. This is accomplished by sending the information with the power required for transmission to the farther of the two nodes. This phase requires the source to use the transmission power:

$$
P_{1}=\max \left(r_{S 1}^{\alpha}, r_{S 2}^{\alpha}\right)
$$

The second and third phases can be described together. These two phases involve sending the information from the first stage to the second stage. They are divided into 
different phases because nodes $R_{3}$ and $R_{4}$ receive the information separately in two different transmissions. These two transmissions could be made using two different frequencies or two different time slots. The required transmission power per phase is: $P_{i}=G \cdot r_{1 i}^{\alpha}+G \cdot r_{2 i}^{\alpha}$, where $i \in 3,4$ and $G$ is the scaling factor of the Alamouti scheme, which is dependent upon the target BER as explained in Chapter 2. Thus, the sum of the power required for both phases can be calculated as:

$$
P_{(2,3)}=G \cdot r_{13}^{\alpha}+G \cdot r_{23}^{\alpha}+G \cdot r_{14}^{\alpha}+G \cdot r_{24}^{\alpha}
$$

The last phase represents the transmission from the two relay nodes in the second stage to the destination using STBC. The transmission power required is:

$$
P_{4}=G \cdot r_{3 D}^{\alpha}+G \cdot r_{4 D}^{\alpha}
$$

Finally, the total transmission power required to send the information from the source to the destination with the double-stage strategy using STBC is:

$$
P^{D-S T B C}=\max \left(r_{S 1}^{\alpha}, r_{S 2}^{\alpha}\right)+G \cdot r_{13}^{\alpha}+G \cdot r_{23}^{\alpha}+G \cdot r_{14}^{\alpha}+G \cdot r_{24}^{\alpha}+G \cdot r_{3 D}^{\alpha}+G \cdot r_{4 D}^{\alpha}
$$

In a wireless network with $N$ relay nodes there could be $N \cdot(N-1)(N-2)(N-3)$ possible combinations of relays acting as $\left(R_{1}, R_{2}\right)$ and $\left(R_{3}, R_{4}\right)$ in two stages as described above. For example, in the case of $N=4$ nodes, there would be 24 possible configurations of four relays carrying out their tasks in a two-stage distributed STBC strategy. 
In summary, in this section three methods of sending data from a source to a destination have been discussed: (i) direct transmission; (ii) store-and-forward transmission; and (iii) distributed STBC in single- and double-stage configurations. Calculations for the total transmit power for each method have also been presented, as well as the number of possible node configurations in a wireless network with a different number of nodes.

\subsection{Equi-Spaced Relay Selection for Single- and Double-Stage Transmission}

In Section 3.1, it has been shown that in the wireless networks under study there are different possible paths which depend upon the positions of the relay nodes. The transmit power can be adjusted and various topologies created which have different power requirements for transmitting the information from the source to the destination. Of interest is the topology and the corresponding power allocation with the minimum total power consumption. An exhaustive search to find the optimum topology is computationally involved. Thus, in this section strategies will be developed to achieve a minimized total power consumption by using less complex searches to arrive at a suitable topology.

In this section the best location for relay nodes will be investigated, to assist in the selection of the best available path in a wireless network. Only the singleand double-stage strategy using the store-and-forward method without STBC will be 
studied.

\subsubsection{Single-Stage Strategy}

First the single-stage strategy will be considered. Figure 3.8 illustrates a colinear source, destination and relay node. In the figure, $r_{S D}$ represents the distance between the source and the destination. The objective is to calculate $x$, the distance between the source and the relay node which will minimize the total power required to transmit data from the source to the destination via the relay node. Rewriting (3.2) for free-space propagation where $\alpha=2$ yields:

$$
P^{S-S F}=x^{2}+\left(r_{S D}-x\right)^{2}
$$

Thus, in order to determine the best location for the relay node so as to minimize the total power required, the derivative of (3.11) is calculated with respect to $x$ and solved for roots. It is found that the minimum power is obtained when $x=\frac{r_{S D}}{2}$, i.e., the optimum relay node location is the equi-spaced position.

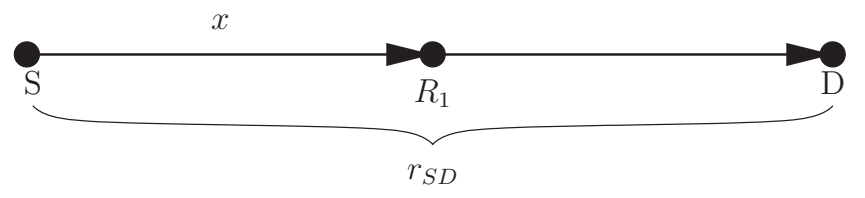

Figure 3.8: A relay node located on the direct path between the source and the destination.

It will now be assumed that the relay node is not co-linear with the source and the destination, as illustrated in Figure 3.9. The distances $r_{S 1}, r_{1 D}$ and $r_{S D}$ represent 
the distances between the source and the node, the node and the destination, and the source and the destination respectively. Assuming that $\alpha=2$, the power required to transmit a signal via the relay node $R_{1}$ is:

$$
P^{S-S F}=r_{S 1}^{2}+r_{1 D}^{2}
$$

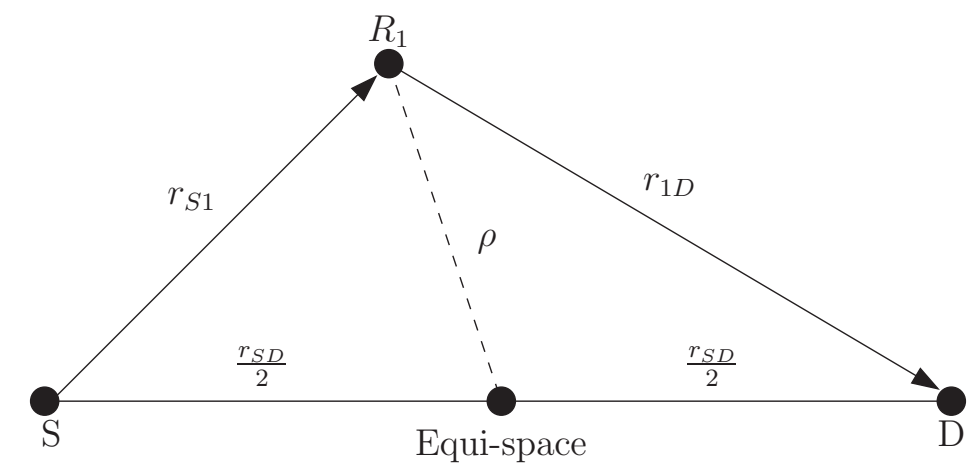

Figure 3.9: Relationship between a relay node and the equi-spaced point.

From the cosine law, the following equation can be derived: $r_{S 1}^{2}=\left(\frac{r_{S D}}{2}\right)^{2}-\rho r_{S D}$. $\cos (\beta)+\rho^{2}$ and $r_{1 D}^{2}=\left(\frac{r_{S D}}{2}\right)^{2}-\rho r_{S D} \cdot \cos (\beta-\pi)+\rho^{2}$ where $\rho$ is the distance between the relay node and the equi-spaced location. Rewriting (3.12) yields:

$$
P^{S-S F}=2\left(\left(\frac{r_{S D}}{2}\right)^{2}+\rho^{2}\right)
$$

Equation (3.13) shows that the closer the relay node is to the equi-spaced point, the less total power is required. Thus, in a network with $N$ relay nodes, the relay node with the smallest $\rho$ is selected. The length $\rho$ represents a cevian in the triangle $S R_{1} D$ as shown in Figure 3.9. It can be calculated using Stewart's theorem:

$$
\frac{r_{S D}}{2}\left(r_{S 1}^{2}+r_{1 D}^{2}\right)=r_{S D}\left(\rho^{2}+\left(\frac{r_{S D}}{2}\right)^{2}\right)
$$


By rewriting (3.14) it can be found that $\rho^{2}$ is:

$$
\rho^{2}=\frac{\left(r_{S 1}^{2}+r_{1 D}^{2}\right)}{2}-\left(\frac{r_{S D}}{2}\right)^{2}
$$

Figure 3.10 illustrates $N$ relay nodes that are randomly distributed. In the case where $\alpha=2$, there are $N$ possible paths for transmitting a signal from the source to the destination, and only one path will be selected. This path is the one that has a relay node with the smallest $\rho_{i}^{2}$ where $i \in 1, \cdots, N$.

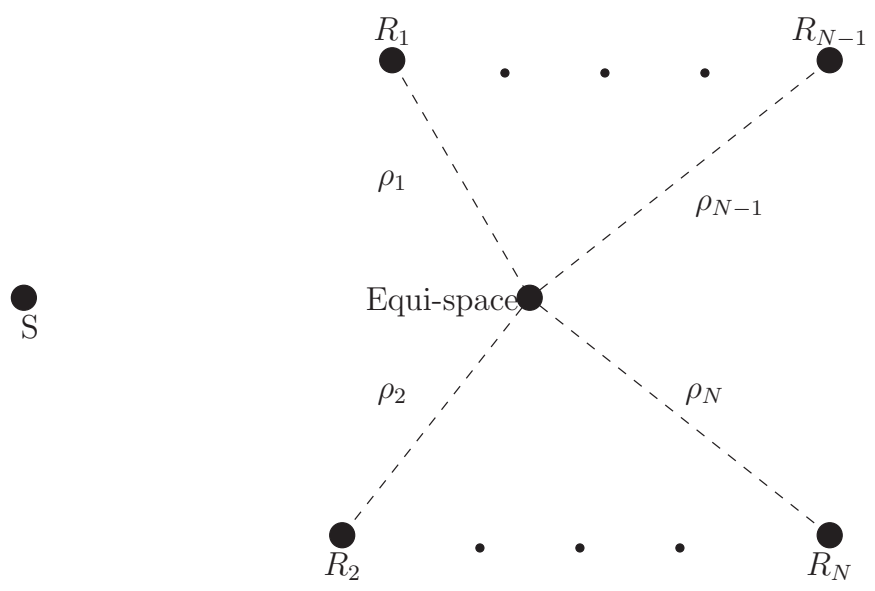

Figure 3.10: Determining the relay node closest to the equi-spaced location.

\subsubsection{Double-Stage Strategy}

In this section the best location for the relay nodes is found for the case where two relay nodes are used to configure a double-stage strategy. In Figure 3.11 the distance between the source and the destination is known and the objective is to calculate the distances $x$ and $y$, which are the distance from the source to the first 
relay node $R_{1}$ and the distance from the first relay node $R_{1}$ to the second relay node $R_{2}$, respectively. From Section 3.1.2.2 it is known that the transmit power required is $P^{D-S F}=x^{2}+y^{2}+\left(r_{S D}-x-y\right)^{2}$ when $\alpha=2$. By solving for roots of the partial derivatives with respect to $x$ and $y$, it is found that the best positions for minimizing the total transmit power are: $x=\frac{r_{S D}}{3}$ and $y=\frac{r_{S D}}{3}$, which are the equi-spaced points on the direct path between the source and the destination.

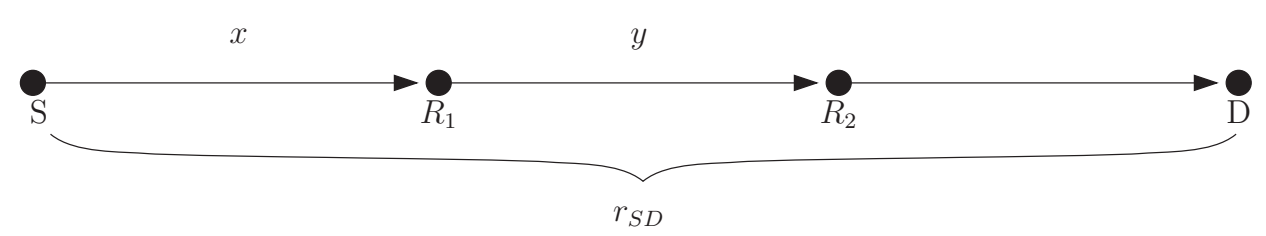

Figure 3.11: Determining optimum point location for the double-stage strategy.

The case will now be considered where the two relay nodes are not located on the direct path between the source and the destination, as illustrated in Figure 3.12. From (3.3) it is known that the total transmission power when $\alpha=2$ is:

$$
P^{D-S F}=r_{S 1}^{2}+r_{12}^{2}+r_{2 D}^{2}
$$

By using the distances $\rho_{i}$ where $i \in 1,2$, which represent the cevians to the equi-spaced points for triangles $S R_{1} D$ and $S R_{2} D$ respectively, and by using their coordinates $\left(x_{i}, y_{i}\right),(3.16)$ can be rewritten as:

$$
\begin{aligned}
P^{D-S F} & =\frac{r_{S D}^{2}}{3}+2\left(x_{1}^{2}+x_{2}^{2}+y_{1}^{2}+y_{2}^{2}\right)+2\left(x_{1} x_{2}-y_{1} y_{2}\right) \\
& =\frac{r_{S D}^{2}}{3}+2\left(\rho_{1}^{2}+\rho_{2}^{2}\right)+2\left(x_{1} x_{2}-y_{1} y_{2}\right)
\end{aligned}
$$




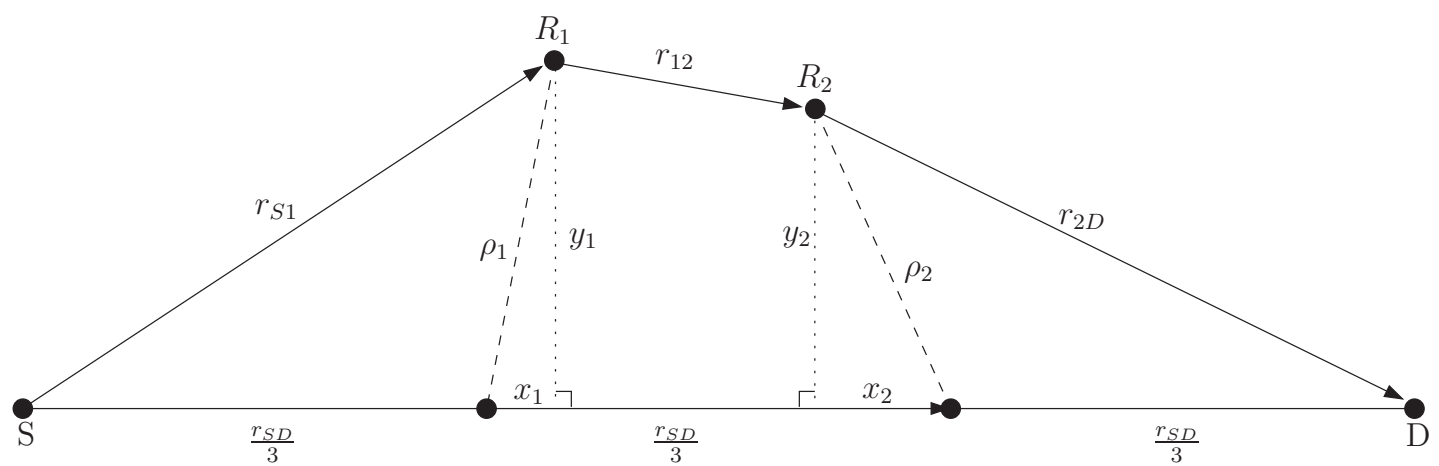

Figure 3.12: Relationship between the equi-spaced locations and the relay nodes.

It can be seen that the second part of the equation $\left(x_{1}^{2}+x_{2}^{2}+y_{1}^{2}+y_{2}^{2}\right)$ will always yield a positive value. However, the last part of the equation will depend upon the position of the two relay nodes and upon how close they are to one another. If it is assumed that both relay nodes have the same sign for the y coordinates, by using Muirhead's inequality it can be proved that $y_{1}^{2}+y_{2}^{2} \geq y_{1} \cdot y_{2}$; therefore any increase in the $y$ coordinates will always increase the total power consumption. Similarly, for the $x$ coordinates $x_{1}^{2}+x_{2}^{2} \geq x_{1} \cdot x_{2}$. From this it can also be proved that $\rho_{1}^{2}+\rho_{2}^{2}=y_{1}^{2}+y_{2}^{2}+x_{1}^{2}+x_{2}^{2} \geq x_{1} x_{2}-y_{1} y_{2}$; this is only when the same coordinates carry the same sign. Since both $x$ and $y$ coordinates could carry different signs, in this case the above proof will not be valid. It is assumed that selecting the relay nodes with the smallest length $(\rho)$ will yield the minimum power consumption. This approach was simulated in [28], and was compared to the exhaustive search method for finding the best relay nodes. It was found that from $46 \%$ to $60 \%$ of the time the preferred relay nodes were not selected, however this caused an increase in power consumption 
of only $0.8 \%$ to $20 \%$ over the minimum value, as compared with $77 \%$ to $99.73 \%$ power savings achieved in comparison to direct transmission [28].

As in Figure 3.12, $\rho_{1}$ and $\rho_{2}$ represent cevians in the triangles $S R_{1} D$ and $S R_{2} D$ respectively. Thus, from these triangles $\rho_{1}$ and $\rho_{2}$ can be calculated by using the equation:

$$
\rho_{i}^{2}=\frac{\left(2 r_{S i}^{2}+r_{i D}^{2}\right)}{3}-\frac{2}{9} r_{S D}^{2}
$$

So, for $N$ relay nodes there could be $N \cdot(N-1)$ possible paths. For all nodes, $\rho_{1}$ and $\rho_{2}$ is calculated by using (3.18). The relay node with the lowest $\rho_{1}$ will be selected to represent the first stage and the relay node with the lowest $\rho_{2}$ will be selected to represent the second stage. In some cases the same relay node might be selected for both the first and second stage. When this occurs it means that using a single-stage strategy is more beneficial.

\subsection{Power Allocation and Optimal Positioning of Relays for STBC Relaying}

Due to the energy benefits of using the relay nodes close to the equi-spaced points in the case of both single- and double-stage strategies using store-and-forward transmission, here the same approach is used to select the relay nodes for single- and double-stage forwarding with distributed STBC. 


\subsubsection{Power Allocation in a Single-Stage Strategy}

First the node selection method for a single-stage strategy is elaborated, following the same approach as in Section 3.2. As illustrated in Figure 3.13, two relay nodes lie on the path between the source and the destination. The objective is to find $x$, which is the distance between the source and the optimum point location that guarantees the highest savings in the total transmission power. The equation (3.6) can be rewritten as follows:

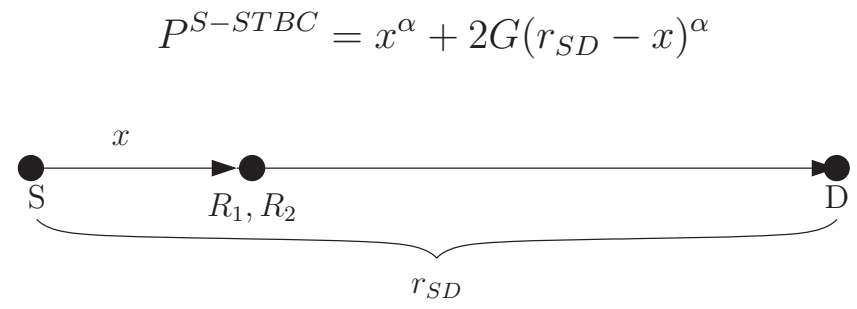

Figure 3.13: Determining the location of the optimum point in a single-stage strategy using STBC.

To find the minimum $P^{S-S T B C}$, the derivative of the right-hand side of (3.19) is

calculated with respect to $x$ and solved for its root. This yields $x=\frac{r_{S D} \sqrt[\alpha-1]{2 G \cdot \alpha}}{\sqrt[\alpha-1]{\alpha}+\sqrt[\alpha-1]{2 G \cdot \alpha}}$. In free space propagation conditions when $\alpha=2$, for a single-stage forwarding mode, the optimum position for relays is $x=\frac{2 G \cdot r_{S D}}{1+2 G}$. Thus, when $G$ decreases $x$ decreases. In other words if the target BER is increased, the scaling factor decreases, which means that $x$ also decreases, bringing the optimum point location closer to the source. In the case where $G=0.1$, as $\alpha$ increases, the distance $x$ also increases, taking the optimum point away from the source. 
So it is found that the optimum point location is not at the equi-spaced position, because the location changes depending upon the target BER, which affects the scaling factor and the attenuation.

The situation where the relay nodes are not located on the direct path between the source and the destination will now be considered, as illustrated in Figure 3.14. The equation (3.6) can be rewritten by selecting $R_{1}$ and $R_{2}$ the two participant relay nodes. When $\alpha=2$ and $G=0.1$, then $P^{S-S T B C}=\max \left(r_{S 1}^{2}, r_{S 2}^{2}\right)+0.1 r_{1 D}^{2}+0.1 r_{2 D}^{2}$.

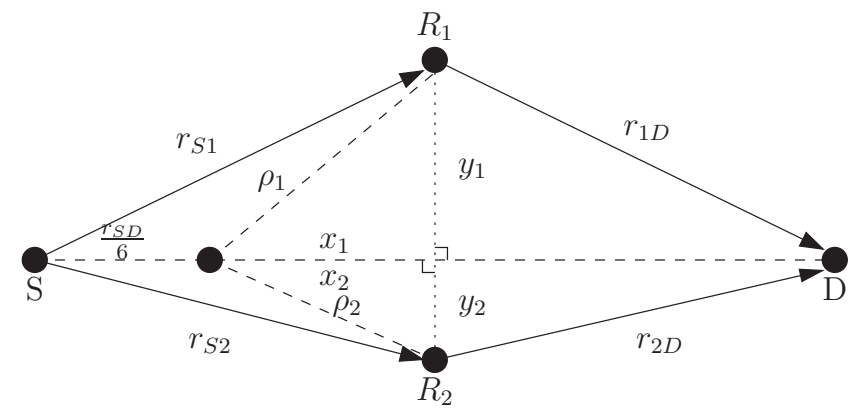

Figure 3.14: Demonstrating the relationship between the relay nodes and the optimum points.

By using the $x_{i}$ and $y_{i}$ coordinates and $\rho_{i}$, which is a cevian in the triangle $S R_{i} D$ where $i \in 1,2$, and assuming that $r_{S 1}$ is larger than $r_{S 2}$, the power equation can be rewritten as follows:

$$
\begin{aligned}
P^{S-S T B C} & =0.3 r_{S D}^{2}+1.1 x_{1}^{2}+1.1 y_{1}^{2}+0.1 x_{2}^{2}+0.1 y_{2}^{2}+\frac{1}{6} r_{S D}\left(x_{1}-x_{2}\right) \\
& =0.3 r_{S D}^{2}+1.1 \rho_{1}^{2}+0.1 \rho_{2}^{2}+\frac{1}{6} r_{S D}\left(x_{1}-x_{2}\right)
\end{aligned}
$$

Beginning with the $y$ coordinates, from (3.20) it can be seen that any increase in the value of $y$ results in an increase in the total transmit power. In the case of the 
$x$ coordinates, a direct relationship with the power consumption cannot be proved mathematically, due to the same problem as in section 3.2.2. For the store-andforward method, it was seen that although it could not be proved mathematically, previous research demonstrated that selecting the relay node closest to the optimum point location is a very effective approach. For this reason the relay nodes that has the smallest cevian length is selected. In Chapter 4 this method is simulated and compared with the exhaustive search method to determine the efficiency of this approach.

\subsubsection{Power Allocation in a Double-Stage Strategy}

In this section the study is extended to the double-stage strategy, and an efficient way to select the relay nodes is investigated. The first step is to determine the optimum point location. As shown in Figure 3.15 it is assumed that $R_{1}$ and $R_{2}$, located on the direct path between the source and the destination, represent the first stage of the transmission, and that $R_{3}$ and $R_{4}$ represent the second stage of the transmission. Here the two relay nodes that are in the same stage are located in the same position; this is done only to find the best location that guarantees the greatest power savings. From Section 3.1.3.2 it is known that the total transmission power is:

$$
P^{D-S T B C}=x^{2}+4 G y^{2}+2 G\left(r_{S D}-y-x\right)^{2}
$$


Thus, by solving the two derivative functions with respect to $x$ and $y$ it can be found that $x=\frac{r_{S D} \sqrt[\alpha-1]{4 G}}{1+\sqrt[\alpha-1]{2}+\sqrt[\alpha-1]{4 G}}$ and $y=\frac{r_{S D}}{1+\sqrt[\alpha-1]{2}+\sqrt[\alpha-1]{4 G}}$, where $x$ is the distance between the source and the first optimum point and $x+y$ is the distance between the source and the second optimum point. It should be noted that the two optimum points depend upon the attenuation and the scaling factor in the same way as in the single-stage strategy. If the attenuation increases, the optimum points will move farther from the source, whereas if the scaling factor increases the optimum points will move closer to the source.

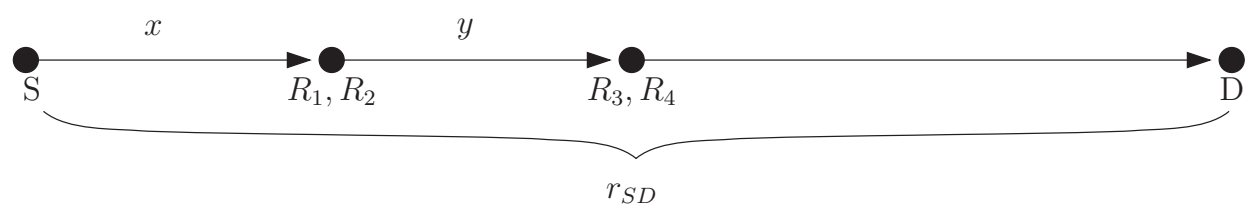

Figure 3.15: Determining the location of the optimum points in a double-stage strategy using STBC.

The case where the relay nodes are located away from the optimum point will now be considered. The same approach is used as in the previous section. The two cevian lengths $\rho_{1 i}$ and $\rho_{2 i}$ are calculated with respect to the two optimum points in the triangles $S R_{i} D$ where $i \in 1,, N$ in a network with $N$ randomly distributed relay nodes. The two nodes with the lowest $\rho_{1 i}$ will be selected to represent the first stage and the two relay nodes with the lowest $\rho_{2 i}$ will be selected to represent the second stage.

In some cases, it is possible that the same node could be selected twice, to represent 
the first and second stage, if it has one of the two lowest cevian lengths. In this situation the transmission path is as shown in Figure 3.16, where the source broadcasts the information to both nodes in the first stage. Here only one transmission takes place instead of the two described in Section 3.1.3.2, because one of the relay nodes from the second stage has already received the information from the source directly. Thus, two relay nodes will forward the information to the relay node in the second stage. Finally, the two relay nodes in the second stage forward the information to the destination.

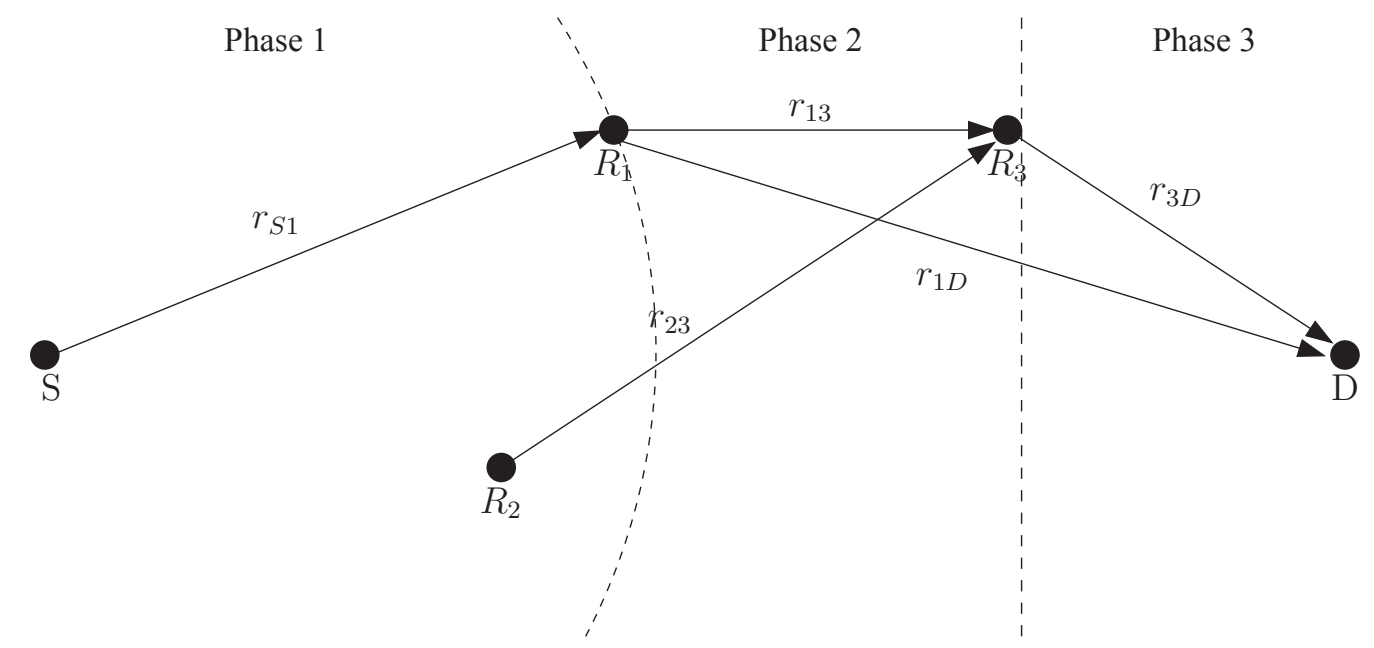

Figure 3.16: Transmitting data where a relay node has been selected in the first and second stage. 


\section{Chapter 4}

\section{Performance}

This chapter demonstrates the power savings of the proposed approach for selecting the relay nodes with the use of STBC in both single- and double-stage transmission strategies. The performance of the proposed approach for selection of the location of the STBC and the forwarding nodes is compared to three other methods. The first method is used for comparing the distributed STBC with the exhaustive search for selecting the most appropriate relay nodes with the lowest total transmission power. The second method is store-and-forward transmission with the use of the exhaustive search. The last method is standard direct transmission. All of the comparisons use the total transmit power as the performance metric. Normalized power savings are also shown with respect to the power used in direct transmission.

This chapter is organized as follows. First, the simulation setup is explained in 
Section 4.1. Then the results concerning excess power consumption in comparison to the exhaustive search method are presented in Section 4.2. The performance of the proposed approach with a good estimate of the channel is presented in Section 4.3, while the performance of the approach with a poor estimate of the channel is described in Section 4.4. Finally, the histogram results of the simulation are shown in Section 4.5.

\subsection{Simulation Setup}

Simulations were implemented with the use of MATLAB, in order to calculate the total transmit power for all of the communication schemes discussed in the previous chapter. The primary focus is on the total transmit power, with perfect knowledge of the power required to reach the nodes from each of the other nodes. These transmit powers depend upon the distances between the randomly distributed nodes and upon the path loss exponent $\alpha$, which is assumed to be the same in the underlying network. The total power results are analyzed: (i) for different propagation conditions; (ii) for different numbers of relay nodes, from $N=10$ to $N=100$ in increments of 10; and (iii) for different sizes of area covered by the wireless network, i.e., for different node densities.

The wireless network nodes in the simulations are assumed to be located in a rectangular area of size $A \times A$. The network consists of the source, the destination 
and the $N$ relay nodes. The source is located at the fixed location $(0,0)$ and the destination is located at the coordinates $(A, A)$. The $N$ relay nodes are randomly distributed in the rectangular area, and the total transmission power for all of the methods is calculated for the given distances between the nodes. Note that it assumed that the power of the noise is $4 \mathrm{~mW}$ at every node. This resulted conveniently in no scaling factor in (1.2) for transmit power when the single-hop communication in Rayleigh fading channel using SISO was considered and the targeted $\mathrm{BER}=10^{-3}$ required $\mathrm{SNR}=24 d B$ with a received signal power equal to $1 \mathrm{~W}$. Usually, the noise levels in practical receivers are lower and the corresponding scaling of the results for the total transmit power presented in this thesis is required as determined by the ratio of the actual noise level over $4 \mathrm{~mW}$. When the normalized power excess is presented in percent in the next sections, the results do not need to be scaled.

The process for random positioning of the nodes and calculation of the power is repeated multiple times (1000) to obtain an average power performance.

\subsection{Excess Power Consumption}

In Section 3.3 it could not be proved analytically that selecting the relay node closest to the "optimum" point location proposed in this thesis would assure maximum power savings, however strong arguments were provided that this is really the case, at least for co-linear nodes or two nodes placed at the same location. This 
section examines how close the total power obtained by this approach is to the total power resulting from an exhaustive search. The simulations were conducted for different propagation environments, different numbers of relay nodes, and different node densities.

First, the selection approach was tested for environments with different path loss exponents $\alpha$. Three types of propagation were used: (i) free space propagation where $\alpha=2$; (ii) ground wave propagation where $\alpha=4$; and (iii) propagation in a dense urban environment where $\alpha=6$. Figure 4.1 shows the percentage of excess transmission power consumption with the use of a single- and double-stage strategy for different numbers of nodes in a network area of $25 \times 25$. It is clear from the figure that as $\alpha$ increases, the excess power consumption resulting from this simplified scheme for node selection is not very high in comparison to the power consumption obtained by the exhaustive search.

However, with the double-stage strategy power efficiency losses are higher, especially for lower node densities and higher values of $\alpha$. The difference in power performance as measured by the percentage of excess power consumption for different values of $\alpha$ decreases as the number of nodes increases. Overall, the loss of power efficiency when using the proposed scheme is not that high when one consider reduction in the computational complexity of the algorithm selecting the topology.

The effect of different numbers of potential relay nodes on the transmission power 


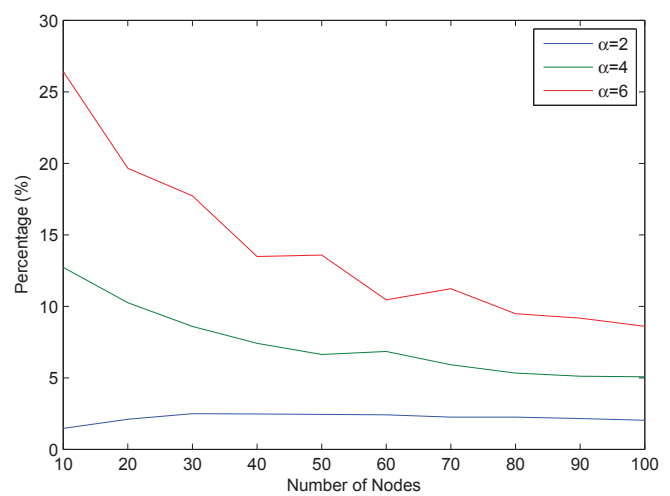

(a)

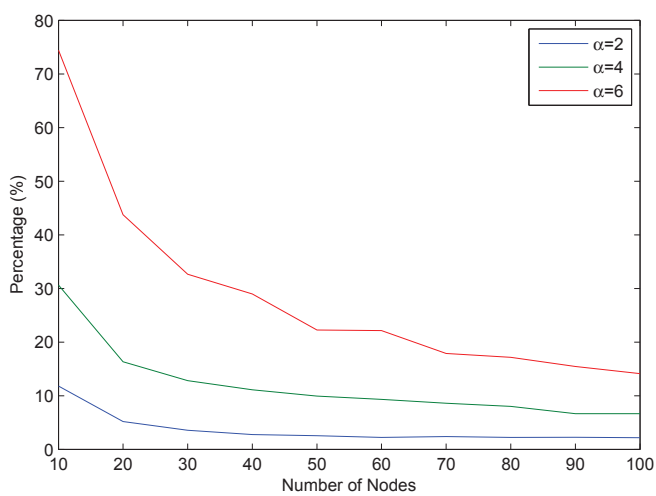

(b)

Figure 4.1: Excess power consumption in comparison with the exhaustive search method for an area of $25 \times 25$ using (a) single-stage and (b) double-stage transmission.

in the proposed approach was then examined. From Figure 4.1 it can be seen that in general, with an increasing number of relay nodes the percentage of excess power consumption decreases. The percentage usually decreases rapidly until the number of relay nodes reaches 50 , after which the percentage begins to stabilize. However, in the case of free space propagation with the single-stage strategy, it can be seen that the lowest percentage is $1.5 \%$ when 10 relay nodes are used; this value increases to $2.5 \%$ and then decreases to $2 \%$ when 100 relay nodes are used.

Finally, the performance results for the selection approach were investigated for different network area sizes. Three different sizes were considered: $25 \times 25,50 \times 50$ and $100 \times 100$. The percentage of excess transmit power consumption in comparison to the power consumption obtained by the exhaustive search method is shown in Figures 4.1, 4.2 and 4.3 for the three different area sizes. Graphs (a) and (b) show the results 
for different values of $\alpha$ with a single- and double-stage strategy, respectively. It can be seen that the curve behavior for the three network sizes is almost the same for a given STBC strategy. The main difference in the results for the three network sizes is in the percentage of excess power consumption when 10 relay nodes are used in the double-stage strategy with a dense urban environment; from the figures it can be seen that the percentage of excess power consumption increases as the network size increases.

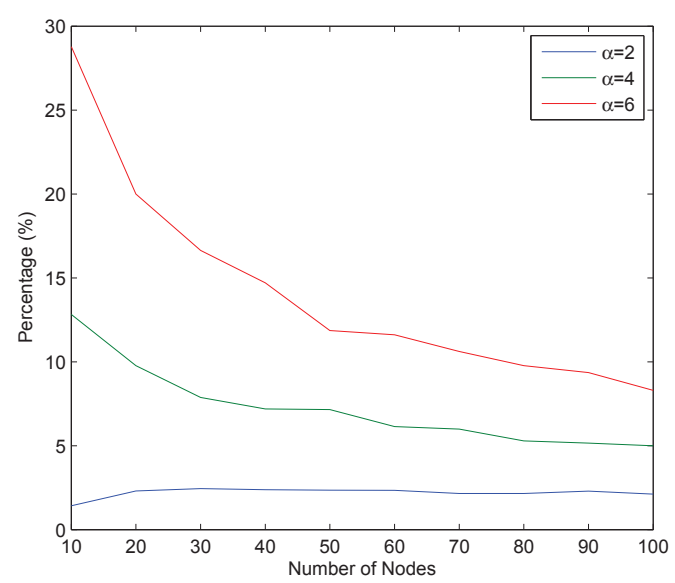

(a)

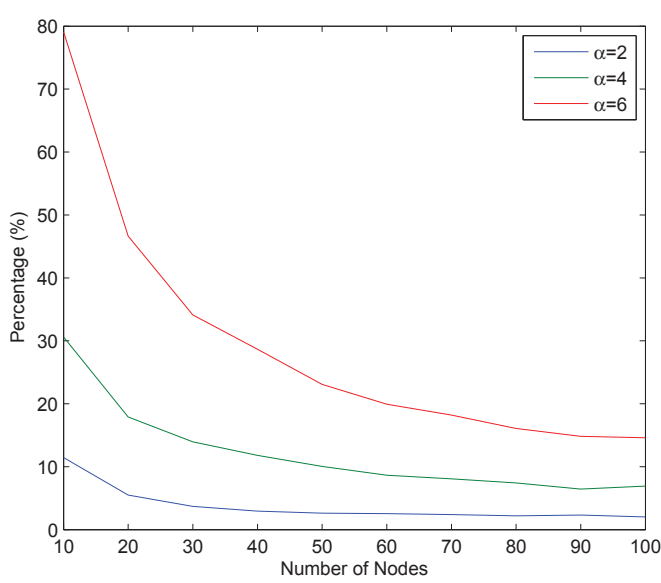

(b)

Figure 4.2: Excess power consumption in comparison with the exhaustive search method for an area of $50 \times 50$ using (a) single-stage and (b) double-stage transmission.

\subsection{Results with Good Channel Estimation}

In this section the simulation results are presented under the assumption of good channel state information (CSI). In the simulations the relay nodes were distributed 


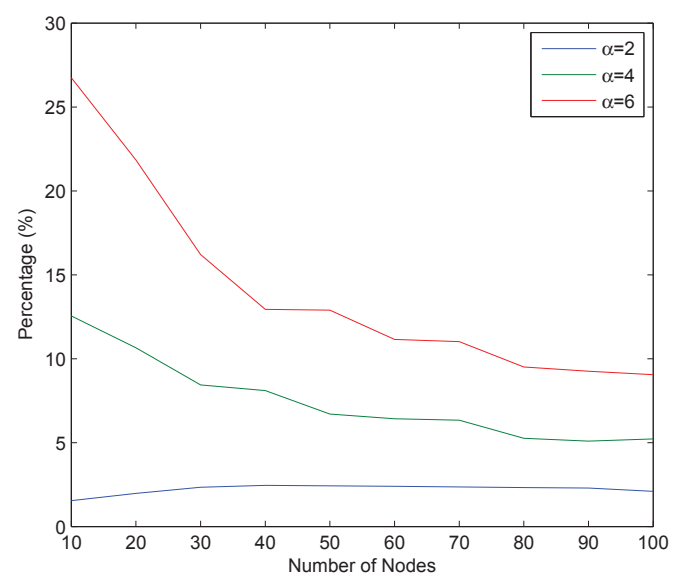

(a)

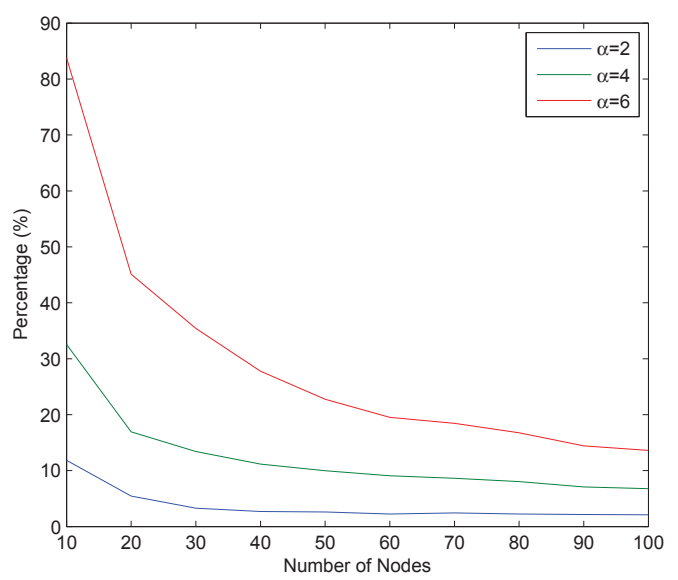

(b)

Figure 4.3: Excess power consumption in comparison with the exhaustive search method for an area of $100 \times 100$ using (a) single-stage and (b) double-stage transmission.

in areas with three different sizes: $25 \times 25,50 \times 50$ and $100 \times 100$. Simulations are shown for numbers of nodes ranging from 10 to 100 in increments of 10 .

For the area with size $25 \times 25$, the graphs in Figure 4.4 show the total transmit power consumption for five methods: (i) direct transmission $(D)$; (ii) store-andforward transmission using a single-stage strategy $(S-S F)$; (iii) store-and-forward transmission using a double-stage strategy $(D-S F)$; (iv) STBC using a single-stage strategy $(S-S T B C)$; and (v) STBC using a double-stage strategy $(D-S T B C)$. The graphs are arranged so that the three rows correspond to the three different propagation environments represented by $\alpha=2,4$ and 6 . The graphs on the left show the power values achieved, while those on the right show the normalized power with respect to the power consumption obtained for direct mode transmission. 
With free space propagation, the transmission power consumed with the use of STBC is the lowest. It can also be seen that with the use of STBC even the proposed selection approach in the single-stage strategy is better than store-and-forward transmission in the double-stage strategy. In comparison with direct transmission, power savings with the use of STBC are $82.4 \%$ for the single-stage and $87.3 \%$ for the double-stage strategy, as shown in Figure 4.4 (b).

With ground wave propagation and propagation in a dense urban environment, it can be seen that the proposed selection approach with STBC in a double-stage strategy offers the lowest total transmit power. However, the single-stage strategy requires more transmit power than store-and-forward transmission using a double-stage strategy. From Figure 4.4 (d), it can be seen that ground wave propagation yields up to $94.4 \%$ power savings with a single-stage and up to $98.2 \%$ power savings with a double-stage strategy. For propagation in a dense urban environment, power savings of $98.4 \%$ for single-stage and $99.8 \%$ for double-stage transmission were achieved.

It should be noted that in a dense urban environment, store-and-forward transmission achieves better power savings than the proposed selection approach when using only 10 relay nodes. However, as the number of relay nodes increases, the proposed approach achieves greater power savings, as shown in Figure 4.4 (f).

In Figures 4.5 and 4.6 simulation results are presented for a network area of size $50 \times 50$ and $100 \times 100$, respectively. The arrangement of the graphs is the same as 
in Figure 4.4. If the results shown in Figures 4.4, 4.5 and 4.6 are compared, it is evident that the power consumption increases as the distance increases. Graphs (b), (d) and (f) illustrate the percentage power savings achieved in comparison to direct transmission. In the three Figures 4.4, 4.5 and 4.6, it can be seen that as the distance increases, the power savings remain the same.

Table 4.1 summarizes the simulation results for power savings (Sav) and excess transmit power consumption (Exc) for an area of $50 \times 50$, where the number of relay nodes is $N=20,60$ and 100 . The results shown are for distributed STBC with two modes of operation, using single- and double-stage transmission. From the table it can be seen that the excess transmit power increases as the density of the environment increases. Thus, for low values of $\alpha$, the simplified approach for node selection proposed in this thesis yields good results. However, for higher values of $\alpha$ power savings could still be improved, even though acceptable power savings were achieved in comparison to store-and-forward transmission.

Table 4.1: Summary of the simulation results for an area of $50 \times 50$.

\begin{tabular}{|c|c|c|c|c|c|c|c|}
\hline \multirow{3}{*}{$\begin{array}{c}\text { Prop } \\
(\alpha)\end{array}$} & \multirow{3}{*}{ stages } & \multicolumn{6}{|c|}{ Number of Relay Nodes $(N)$} \\
\hline & & \multicolumn{2}{|c|}{20} & \multicolumn{2}{|c|}{60} & \multicolumn{2}{|c|}{100} \\
\hline & & Sav & Exc & Sav & Exc & Sav & Exc \\
\hline \multirow[t]{2}{*}{2} & single & $80.1 \%$ & $2.3 \%$ & $82.1 \%$ & $2.3 \%$ & $82.4 \%$ & $2.1 \%$ \\
\hline & double & $83.6 \%$ & $5.5 \%$ & $86.7 \%$ & $2.5 \%$ & $87.3 \%$ & $2 \%$ \\
\hline \multirow[t]{2}{*}{4} & single & $93 \%$ & $9.7 \%$ & $94.1 \%$ & $6.1 \%$ & $94.4 \%$ & $5 \%$ \\
\hline & double & $97.3 \%$ & $17.9 \%$ & $98.1 \%$ & $8.6 \%$ & $98.2 \%$ & $6.9 \%$ \\
\hline \multirow[t]{2}{*}{6} & single & $97.6 \%$ & $20 \%$ & $98.3 \%$ & $11.6 \%$ & $98.4 \%$ & $8.3 \%$ \\
\hline & double & $99.5 \%$ & $46.6 \%$ & $99.7 \%$ & $19.9 \%$ & $99.8 \%$ & $14.6 \%$ \\
\hline
\end{tabular}




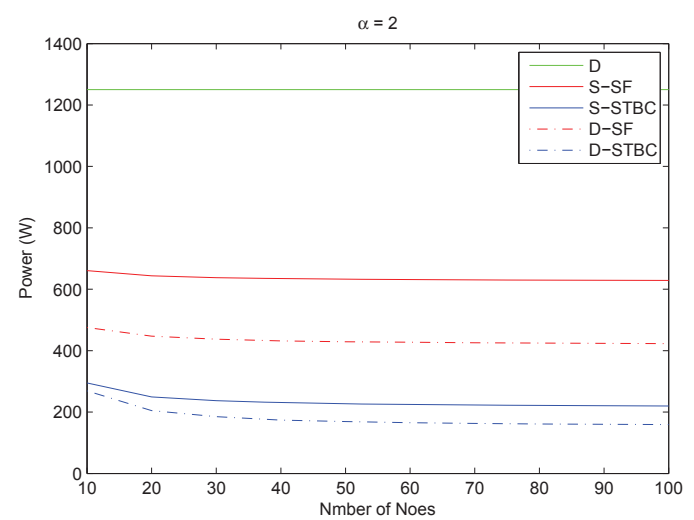

(a)

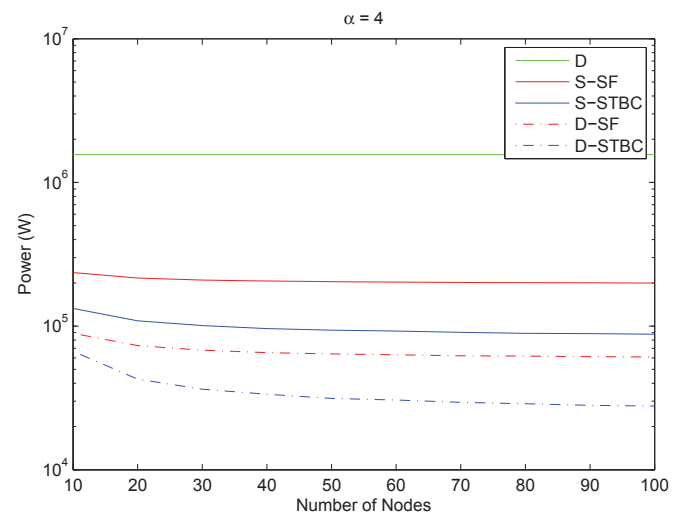

(c)

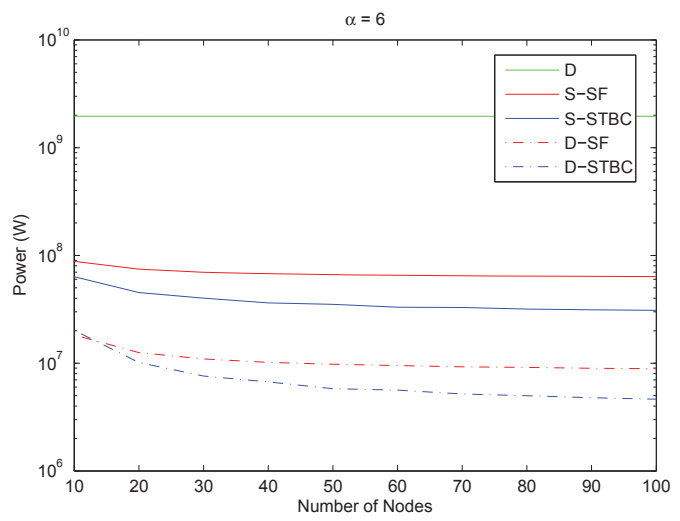

(e)

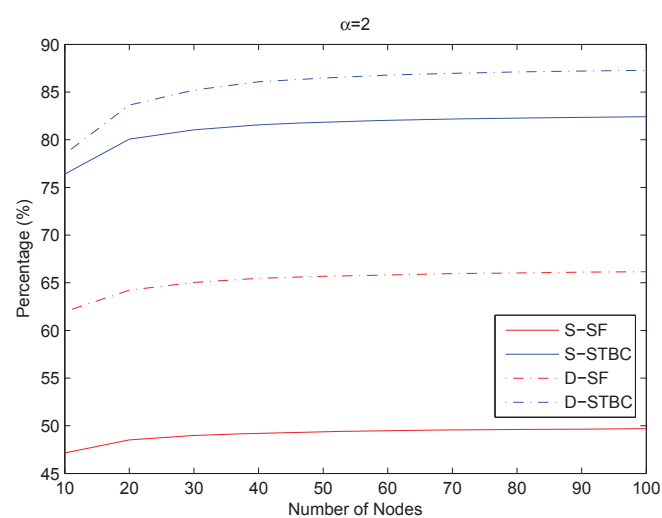

(b)

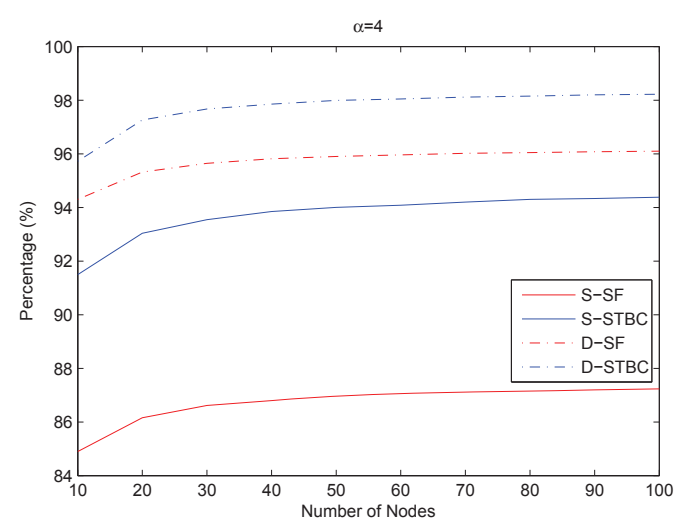

(d)

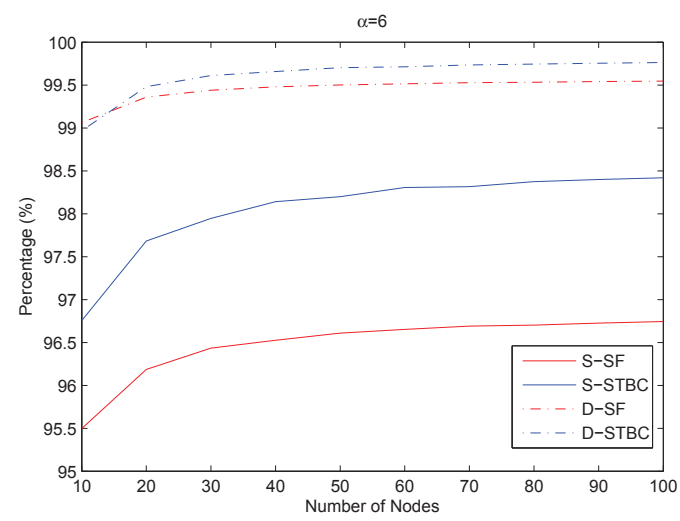

(f)

Figure 4.4: Transmission power consumption and the percentage of power savings in comparison with direct transmission for an area of $25 \times 25$, (a) \& (b) with free space propagation, (c) \& (d) with ground wave propagation, and (e) \& (f) with propagation in a dense urban environment. 


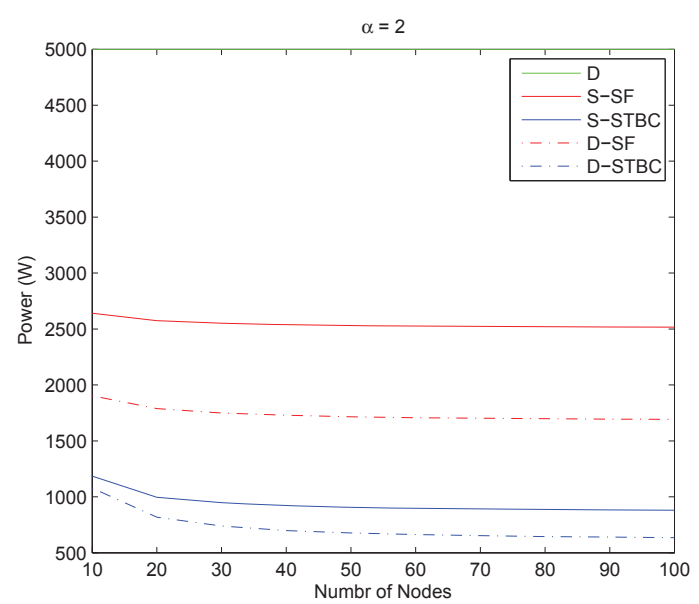

(a)

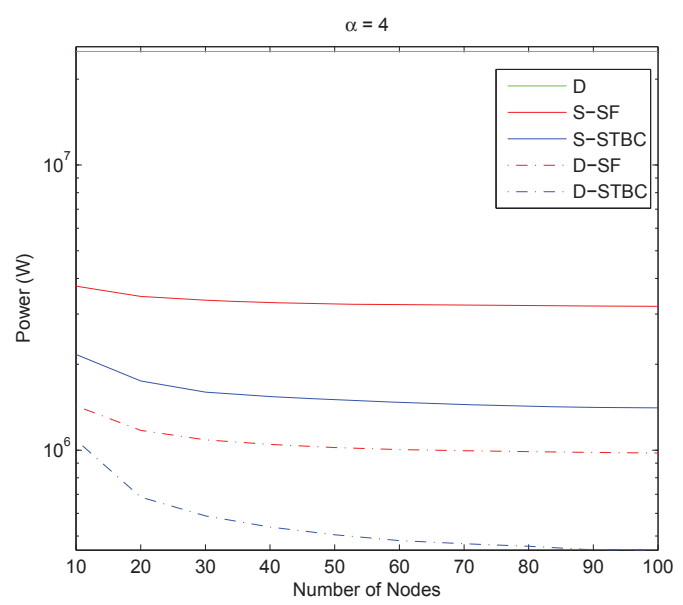

(c)

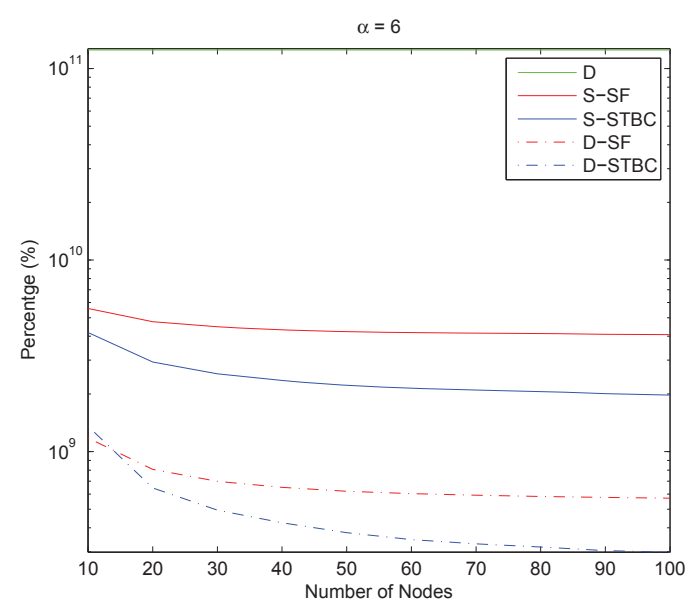

(e)

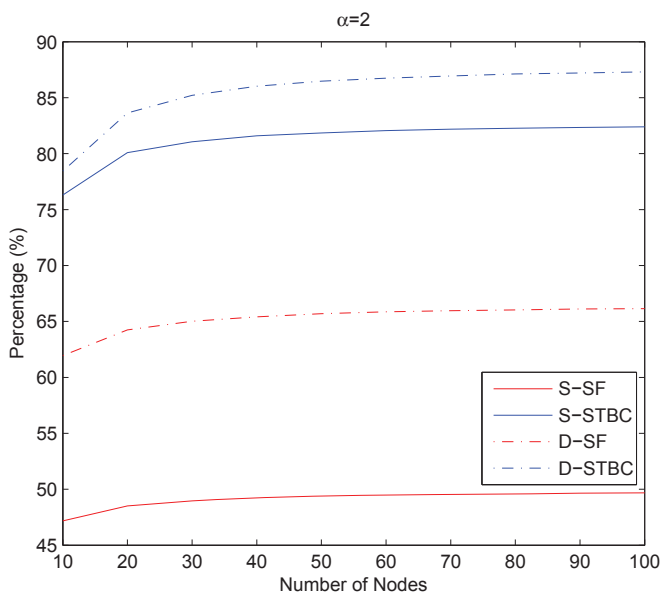

(b)

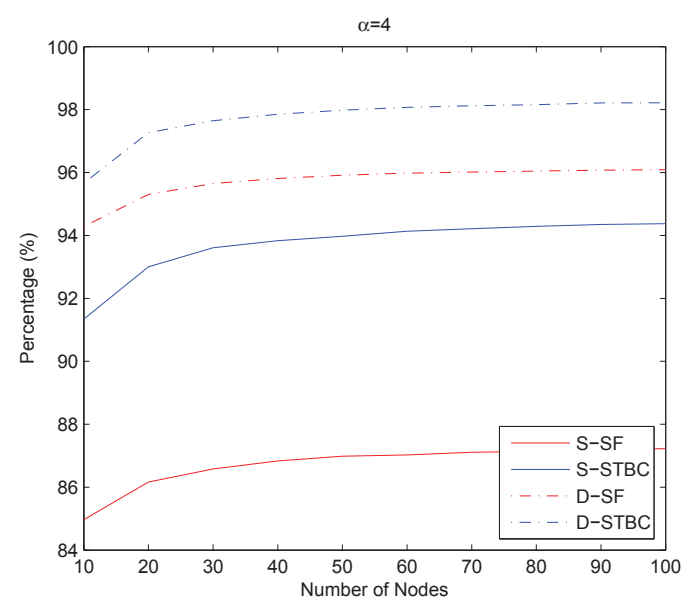

(d)

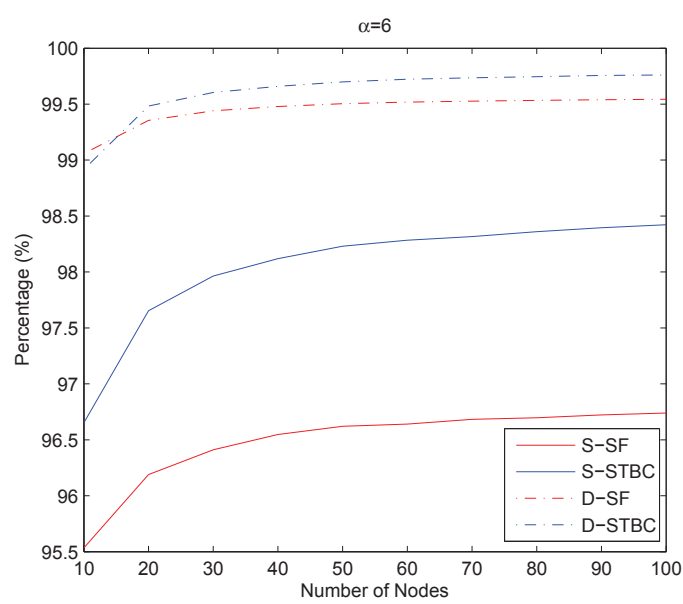

(f)

Figure 4.5: Transmission power consumption and the percentage of power savings in comparison with direct transmission for an area of $50 \times 50$, (a) \& (b) with free space propagation, (c) \& (d) with ground wave propagation, and (e) \& (f) with propagation in a dense urban environment. 


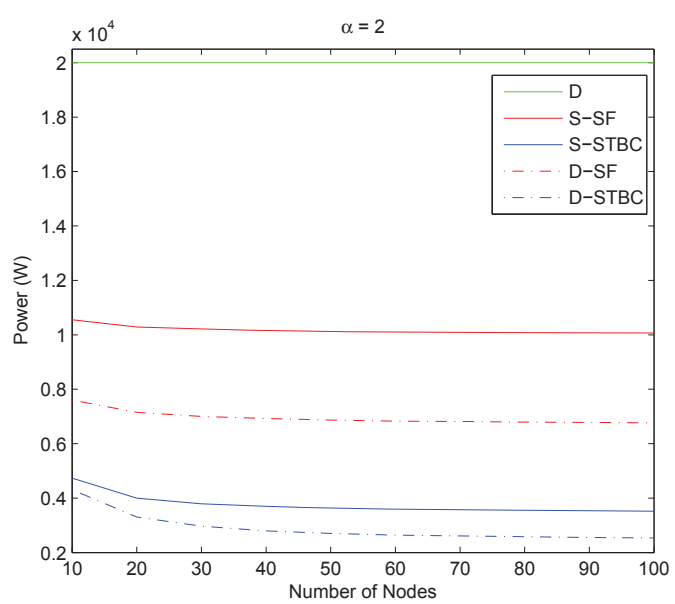

(a)

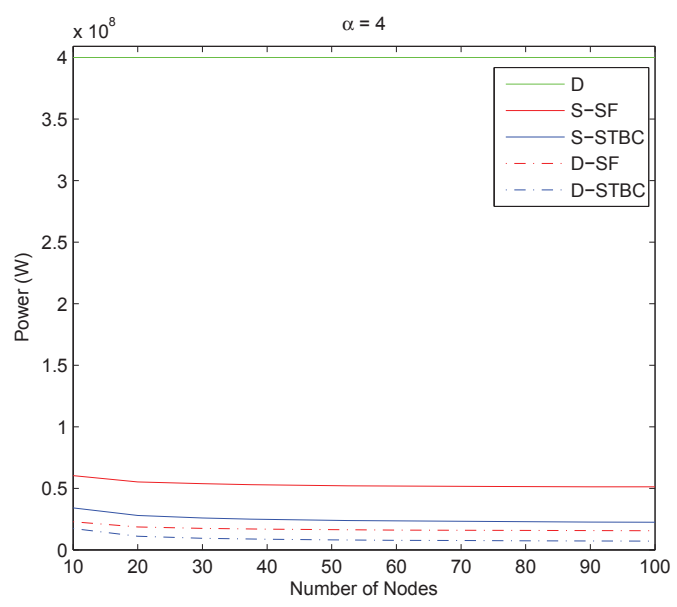

(c)

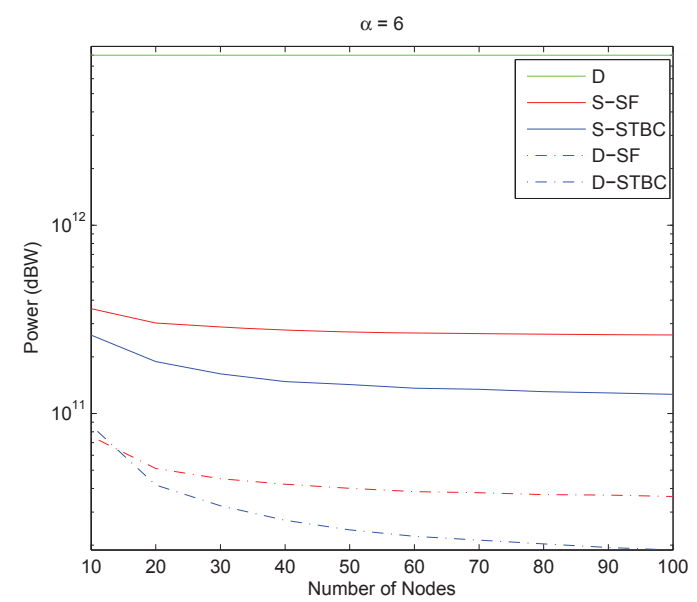

(e)

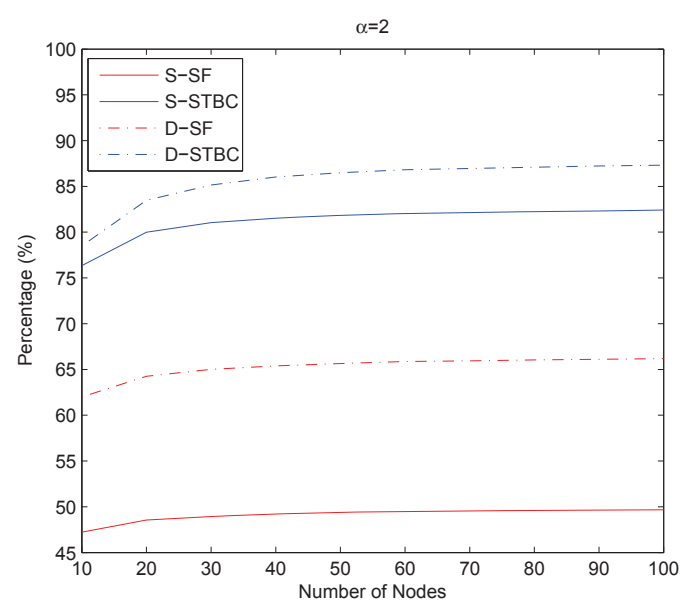

(b)

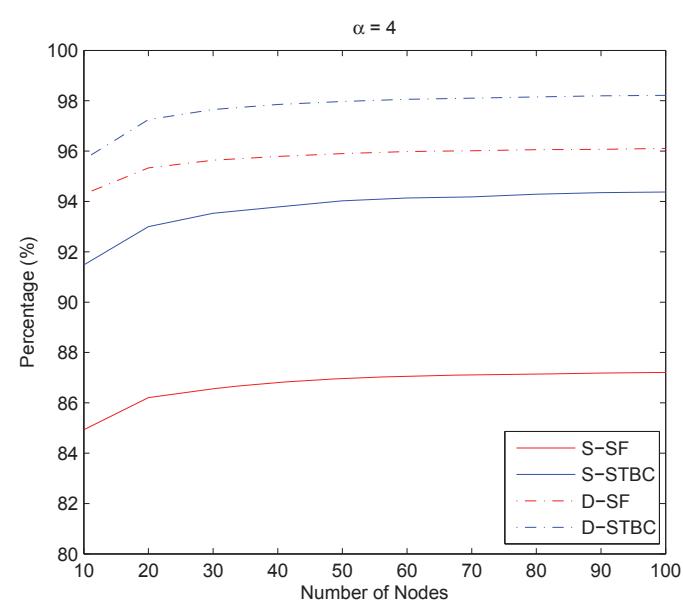

(d)

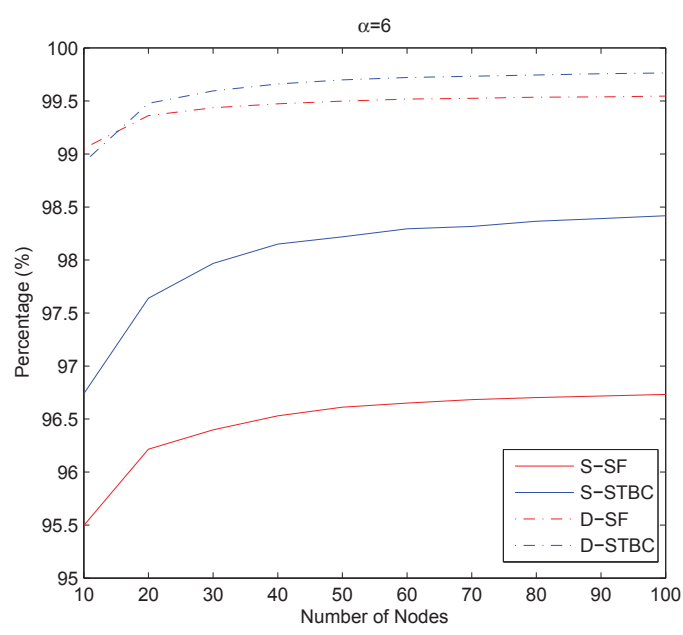

(f)

Figure 4.6: Transmission power consumption and the percentage of power savings in comparison with direct transmission for an area of $100 \times 100$, (a) \& (b) with free space propagation, (c) \& (d) with ground wave propagation, and (e) \& (f) with propagation in a dense urban environment. 


\subsection{Results with Poor Channel Estimation}

In this section it is assumed that the CSI is incorrectly estimated, and that an accurate value for $\alpha$ is not supplied to the algorithm for selecting the relay nodes. This incorrect estimation leads to incorrect calculation of the optimum relay location and thus results in the selection of relay nodes that are not optimal for minimizing the power consumption. To demonstrate the results of an incorrect estimation, two scenarios are considered. Here only one network size is considered because, as seen in the previous section, the percentage of power savings is not affected by network size.

In the first scenario it is assumed that a dense urban environment is incorrectly estimated as a free space environment. Figure 4.7 shows the percentage of power savings in comparison to direct transmission when the incorrect estimate is used. From this figure it can be seen that, in comparison to Figure 4.6(f), the power savings are reduced by only $0.8 \%$ in the double-stage strategy and $5.4 \%$ in the single-stage strategy. Although the loss of power efficiency is not great, it should be noted that if the store-and-forward method was used it would yield higher power savings, because it would not be affected by the poor estimate.

In the second scenario, propagation in a free space environment is incorrectly estimated as a dense urban environment. The results are presented in Figure 4.8. In comparison to Figure 4.6 (b) power savings are reduced by $4.1 \%$ in the double-stage strategy and $8.6 \%$ in the single-stage strategy, which is more than in the first scenario. 


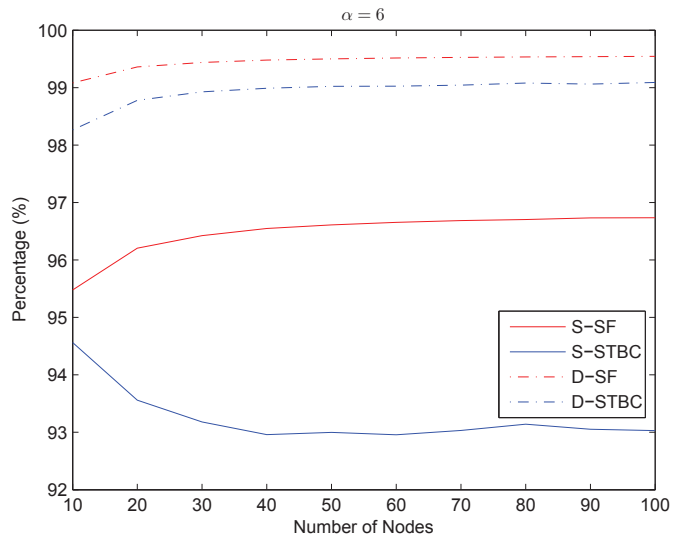

Figure 4.7: The percentage of power savings with an incorrect estimate of propagation in a dense urban environment.

However, it can be seen that even with this incorrect estimate of the channel better power savings are obtained than with the store-and-forward method.

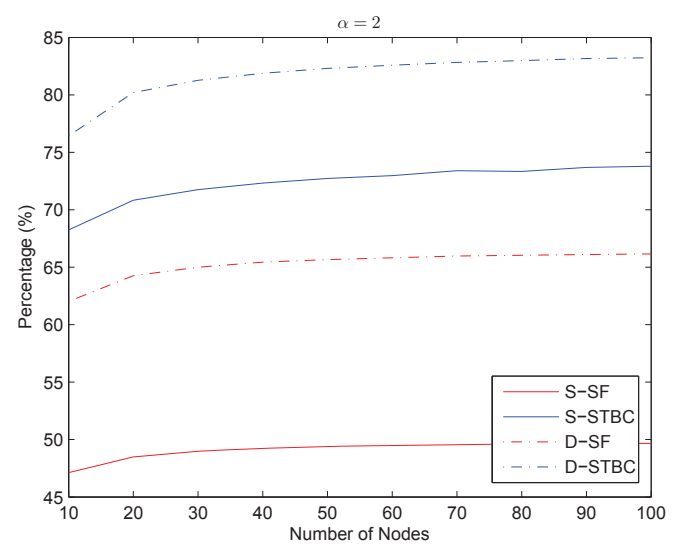

Figure 4.8: The percentage of power savings with an incorrect estimate of free space propagation. 


\subsection{Histograms}

In this section, histograms of the transmit power are presented for 1000 iterations of random placements of 100 nodes in the area of $100 \times 100$. Only ground wave propagation is considered, where $\alpha=4$. The histograms illustrate the distribution of the total transmission power resulted from the topologies with the minimized power in the different iterations (random placement of nodes). Figure 4.9 presents the histogram for a single-stage strategy, while Figure 4.10 shows the histogram for a double-stage strategy with distributed STBC. While in the previous sections, we presented the total transmit power averaged over different placements of nodes, the histograms show that for static placement of nodes the actual power will depend on the nodes positions. Because in Fig. 4.10 the power levels are less spread around the mean then in Fig. 4.9 where the power variability is greater, it is expected that the discussions about power savings based on averaged results will be more consistent for double-stage strategy in comparison to the discussion for single-stage strategy. 


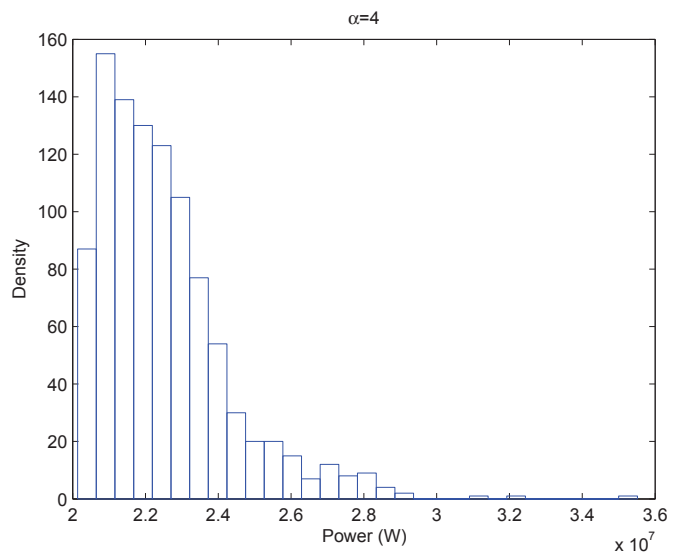

Figure 4.9: Histogram of transmission power consumption for a single-stage strategy in a ground wave environment.

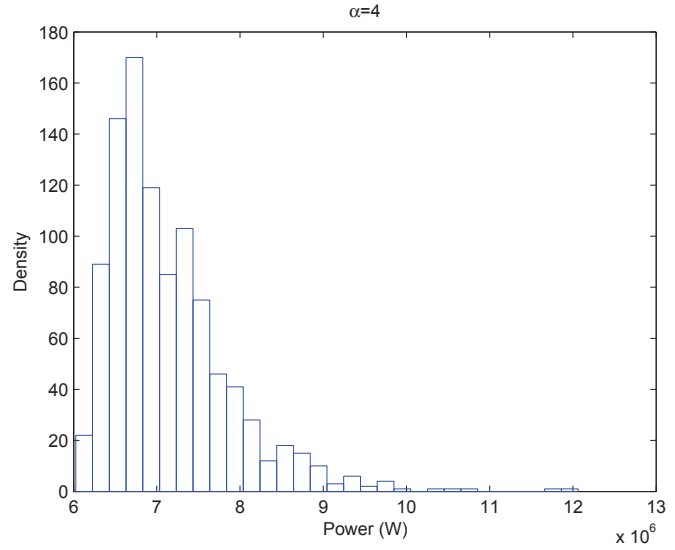

Figure 4.10: Histogram of transmission power consumption for a double-stage strategy in a ground wave environment. 


\section{Chapter 5}

\section{Conclusions and Future Work}

In this chapter, the thesis is summarized and suggestions for future work are discussed. The thesis contributions are reviewed in Section 5.1 and potential further research is suggested in Section 5.2.

\subsection{Thesis Contributions}

The main contribution of this thesis is the development of a method for selection of the relay nodes with a corresponding power allocation to minimize the total transmit power with the distribution of STBC. Two transmission strategies are considered in this thesis: the single-stage strategy and the double-stage strategy in an ad-hoc wireless network. In addition, this thesis calculates the power savings achieved for the thesis approach and compares them with power used in other methods for selecting 
the relay nodes and forwarding the data in different propagation environments and with different numbers of relay nodes.

This work is the first to investigate transmission power savings by integrating the cooperative STBC relaying with adaptive power allocation. Based on the knowledge of the distances between all of the nodes, and by calculating the distance between the relay nodes and the optimum node location, the topology minimizing the total transmit power is selected. In the initial stage, it is assumed that all of the nodes in the wireless network detect each other's signals. Thus, each node measures the level of signal energy to determine the distance and the CSI relative to its neighbors, and then distributes this information to the other nodes in the network to be saved in a common database. When a node is to communicate with a specific destination, it first calculates the optimum relay locations for the transmission and then chooses the appropriate relay nodes by using cevian theory. The required transmit power to maintain the target BER is calculated by using the interdistance and the path loss exponent, which are already available in the database from the initial stage. The proposed approach also takes advantage of the broadcast nature of wireless transmissions, which increases power savings. Specific issues that have been addressed include:

1. Calculating the optimum relay location

This thesis focuses on the total transmit power in both single- and double-stage 
strategies with the utilization of distributed STBC. From this, the optimum location of the relay nodes is found that offers the minimum power consumption. This thesis also describes how the location of the optimum relay point changes depending upon the propagation $\alpha$.

2. Choosing the appropriate relay nodes to minimize transmission power consumption After the optimum point location between a source and a destination has been found, the distances between this point and all other relay nodes are calculated by using cevian theory. The relay nodes with the minimum distances are selected to create the routing path between the source and the destination. This simple approach reduces the processing complexity of obtaining the routing path, in comparison to the use of the exhaustive search algorithm.

\section{Characterizing the performance of the proposed approach}

With the use of simulations it is shown that the selection approach results in reasonable excess transmission power consumption, as compared to the exhaustive search method. Even with the excess total transmit power, it is demonstrated that the thesis approach still generates more power savings than the store-andforward method. It is also shown that power savings are not seriously affected when an incorrect CSI estimation occurs. 


\subsection{Suggested Future Work}

1. Improving the power calculation

In this thesis the adaptive transmission power levels are considered as a continuous range, dependent only upon distance and attenuation. However, in reality most digital transmission devices use a range of discrete transmission power levels. The power control error has also not been considered, as it is caused by the distance and the delay. Thus, there is a need to analyze the effect of the power control error and to investigate how the quantization of the power level affects the proposed relay selection approach.

2. Transmitting without CSI and the distance between antennas

In this thesis the availability of channel state information is assumed. However, most STBC models consider transmitting and receiving without the knowledge of channel state information. Another issue that has not been considered in this thesis is the distance between the two cooperative relay nodes. It is known that fading effects are less as the distance between the cooperative relay nodes increases.

3. Synchronous transmission

In previous chapters it is stated that when the two cooperative relay nodes start transmission this must be done simultaneously. Thus, there should be 
open communication between the two cooperative nodes or feedback from other stages to give direction or status information about the other node. This study does not discuss the method of synchronization between the nodes, and it is assumed that the synchronization is achieved internally. 


\section{Bibliography}

[1] W. Ajib, D. Haccoun, and J. Frigon, "MIMO Link Layer Transmission Techniques Based on Cross Layer Design," Journal of Communications, vol. 2, pp. $28-35,2007$.

[2] W. Jaafar, W. Ajib, and D. Haccoun, "On the Performance of Distributed-STBC in Multi-hop Wireless Relay Networks," European Wireless Conference, pp. 223$230,2010$.

[3] F. E. Oggier and B. Hassibi, "Code Design for Multihop Wireless Relay Networks," Eurasip Journal on Advances in Signal Processing, vol. 2008, pp. 1-12, 2008.

[4] E. Yilmaz and S. G. Kiani, "Transmit Cooperation Versus Distributed Coordination in Interference Links," in IEEE Wireless Communications $\&$ Networking Conference, 2009, pp. 1000-1004.

[5] R. Pabst, B. H. Walke, D. C. Schultz, P. Herhold, H. Yanikomeroglu, M. Lott, W. Zirwas, M. Dohler, H. Aghvami, D. D. Falconer, and G. P. Fettweis, "Relaybased deployment concepts for wireless and mobile broadband radio," IEEE Communications Magazine, vol. 42, pp. 80-89, 2004.

[6] C. T. K. Ng and A. J. Goldsmith, "Transmitter Cooperation in Ad-Hoc Wireless Networks: Does Dirty-Paper Coding Beat Relaying?" IEEE Information Theory Workshop, pp. 227-282, 2004.

[7] Y. Tsai and L. Lin, "Optimal Power Allocation for Decode-and-Forward Cooperative Diversity Under an Outage Performance Constraint," IEEE Communications Letters, vol. 14, pp. 945-947, 2010.

[8] M. O. Hasna and M. slim Alouini, "Optimal power allocation for relayed transmissions over Rayleigh-fading channels," IEEE Transactions on Wireless Communications, vol. 3, pp. 1999-2004, 2004. 
[9] A. Khabbazibasmenj and S. A. Vorobyov, "Power Allocation in Decode-andForward Cooperative Networks via SEP Minimization," 3rd IEEE International Workshop on Computational Advances in Multi-Sensor Adaptive Processing, pp. 328-331, 2009.

[10] J. Luo, R. S. Blum, L. Cimini, L. Greenstein, and A. Haimovich, "Power Allocation in a Transmit Diversity System With Mean Channel Gain Information," IEEE Communications Letters, vol. 9, pp. 616-618, 2005.

[11] L. Zhang and L. Cimini, "Efficient power allocation for decentralized distributed space-time block coding," IEEE Transactions on Wireless Communications, vol. 8, pp. 1102-1106, 2009.

[12] L. Zuari, A. Conti, and V. Tralli, "Effects of Relay Position and Power Allocation in Space-Time Coded Cooperative Wireless Systems," 6th International Symposium on Wireless Communication Systems, pp. 700-704, 2009.

[13] W. Stallings, Data and Computer Communications. Upper Saddle River: Prentice Hall, 2000.

[14] A. Goldsmith, Wireless Communications. New York: Cambridge, 2005.

[15] S. Haykin and M. Moher, Modern Wireless Communications. Upper Saddle River: Prentice Hall, 2005.

[16] R. L. Freeman, Radio System Design for Telecommunicatoins. United States of America: John Wiley and Sons, 1987.

[17] S. Rangappa, "Cocasting and Power Control for Energy Efficient Information Dissemination in WSNs," Master's thesis, Dalhousie University, Halifax, 2010.

[18] P. Santi, Topology Control in Wireless Ad Hoc and Sensor Networks. England: John Wiley and Sons, 2005.

[19] M. Dohler and Y. Li, Cooperative Communicatoins. England: John Wiley and Sons, 2010.

[20] C. S. Murthy and B. S. Manoj, Ad Hoc Wireless Networks Architectures and Protocols. Upper Saddle River: Prentice Hall, 2004.

[21] Y. Akaiwa, Introduction to Digital Mobile Communication. New York: John Wiley and Sons, 1997.

[22] J. G. Proakis, Digital Communications. New York: McGraw-Hill, 2001. 
[23] F. Xiong, Digital Modulation Techniques. Norwood: Artech House, 2000.

[24] B. Vucetic and J. Yuan, Space-Time Coding. England: John Wiley and Sons, 2003.

[25] G. D. Durgin, Space-Time Wireless Channels. Upper Saddle River: Prentice Hall, 2003.

[26] S. Alamouti, "A simple transmit diversity technique for wireless communications," IEEE Journal on Selected Areas in Communications, vol. 16, pp. 14511458, 1998.

[27] M. Jankiraman, Space-Time codes and MIMO Systems. Norwood: Artech House, 2004.

[28] S. H. Melvin and J. Ilow, "Equi-spaced Relay Selection in Co-operative Communication Networks with Power Control," Private Communications. 